\title{
ROBUST QUASI-LPV MODEL REFERENCE FTC OF A QUADROTOR UAV SUBJECT TO ACTUATOR FAULTS
}

\author{
DAMIANO ROTONDO ${ }^{a, *}$, FATIHA NEJJARI $^{a}, \operatorname{ViCENÇ~PUIG~}^{a, b}$ \\ ${ }^{a}$ Automatic Control Department \\ Polytechnic University of Catalonia (UPC), Rambla de Sant Nebridi, 11, 08222 Terrassa, Spain \\ e-mail: damiano.rotondo@yahoo.it, \{fatiha.nejjari, vicenc.puig\} @upc.edu \\ ${ }^{b}$ Institute of Robotics and Industrial Informatics \\ UPC-CSIC, Carrer de Llorens i Artigas, 4-6, 08028 Barcelona, Spain
}

\begin{abstract}
A solution for fault tolerant control (FTC) of a quadrotor unmanned aerial vehicle (UAV) is proposed. It relies on model reference-based control, where a reference model generates the desired trajectory. Depending on the type of reference model used for generating the reference trajectory, and on the assumptions about the availability and uncertainty of fault estimation, different error models are obtained. These error models are suitable for passive FTC, active FTC and hybrid FTC, the latter being able to merge the benefits of active and passive FTC while reducing their respective drawbacks. The controller is generated using results from the robust linear parameter varying (LPV) polytopic framework, where the vector of varying parameters is used to schedule between uncertain linear time invariant (LTI) systems. The design procedure relies on solving a set of linear matrix inequalities (LMIs) in order to achieve regional pole placement and $\mathcal{H}_{\infty}$ norm bounding constraints. Simulation results are used to compare the different FTC strategies.
\end{abstract}

Keywords: linear parameter varying systems, fault tolerant control, quadrotor, model reference-based control, linear matrix inequalities.

\section{Introduction}

In the last years, unmanned aerial vehicles (UAVs) have become an important topic of research because of their characteristics that make them ideal vehicles for several applications, such as security, traffic surveillance, management of natural risks, environment exploration, agriculture and military (Sharifi et al., 2010). Considerable efforts have been made to control these vehicles, applying techniques ranging from PID control (Hoffmann and Waslander, 2008) to nonlinear control techniques (Chowdhary et al., 2014), such as dynamic feedback control (Mokhtari and Benallegue, 2004), backstepping (Aranjo-Estrada et al., 2009; Guenard et al., 2008), nested saturations (Castillo et al., 2005), predictive/nonlinear $\mathcal{H}_{\infty}$ control (Raffo et al., 2010) and quaternion-based feedback for event-triggered stabilization (Guerrero-Castellano et al., 2013).

Recently, some works have considered fault detection and diagnosis (FDD) and fault tolerant control

\footnotetext{
${ }^{*}$ Corresponding author
}

(FTC) for UAVs (Zhang et al., 2013); see Table 1. Generally speaking, FTC techniques can be classified into two types: passive and active (see the works of Zhang and Jiang (2008) as well as Benosman (2010) for reviews). In passive techniques, controllers are fixed and designed to be robust against a class of presumed faults. This approach needs neither FDD schemes nor controller reconfiguration, but it has limited fault-tolerant capabilities. On the other hand, active techniques react to system component failures actively by reconfiguring control actions so that the stability and acceptable performance of the entire system can be maintained. In such control systems, the controller compensates for the impacts of the faults either by selecting a pre-computed control law or by synthesizing a new one on-line. In the last years, some comparative studies between passive and active FTC techniques have appeared (see, e.g., Jiang and Yu, 2012; Rotondo et al., 2013b). A comparison of active and passive FTC strategies shows the importance of investigating the design of hybrid techniques that can merge the benefits of active and passive FTC, while 
Table 1. Techniques applied for fault tolerant control.

\begin{tabular}{|c|c|}
\hline Technique & Reference \\
\hline $\begin{array}{c}\text { model } \\
\text { predictive } \\
\text { control (MPC) }\end{array}$ & $\begin{array}{c}\text { Izadi } \text { et al., } 2010 \\
\text { Izadi } \text { et al., } 2011 \\
\text { Yu et al., } 2013\end{array}$ \\
\hline $\begin{array}{c}\text { feedback } \\
\text { linearization } \\
(\mathrm{FL})\end{array}$ & Freddi et al., 2011 \\
\hline $\begin{array}{l}\text { model reference } \\
\text { adaptive control } \\
\text { (MRAC) }\end{array}$ & $\begin{array}{c}\text { Dydek et al., 2010a } \\
\text { Dydek et al., 2010b } \\
\text { Sadeghzadeh } \text { et al., 2011a } \\
\text { Sadeghzadeh } \text { et al., 2011b } \\
\text { Chamseddine } \text { et al., } 2011\end{array}$ \\
\hline $\begin{array}{l}\text { control } \\
\text { allocation }\end{array}$ & $\begin{array}{c}\text { Zhou } \text { et al., } 2010 \\
\text { Chamseddine } \text { et al., } 2012\end{array}$ \\
\hline $\begin{array}{c}\text { gain-scheduled } \\
\text { PID }\end{array}$ & $\begin{array}{l}\text { Sadeghzadeh } \text { et al., 2011a } \\
\text { Sadeghzadeh } \text { et al., 2011b } \\
\text { Milhim } \text { et al., } 2010 \\
\text { Amoozgar } \text { et al., } 2012\end{array}$ \\
\hline backstepping & $\begin{array}{c}\text { Zhang et al., } 2010 \\
\text { Khebbache et al., } 2012\end{array}$ \\
\hline $\begin{array}{c}\text { sliding } \\
\text { mode } \\
\text { control }(\mathrm{SMC})\end{array}$ & $\begin{array}{l}\text { Sharifi } \text { et al., } 2010 \\
\text { Li } \text { et al., } 2013 \\
\text { Merheb et al., } 2013\end{array}$ \\
\hline $\begin{array}{l}\text { adaptive } \\
\text { control }\end{array}$ & Zhang and Zhang, 2010 \\
\hline
\end{tabular}

reducing or even nullifying their respective drawbacks. Some results in this direction have been obtained in a few papers that have appeared recently (e.g., Maki et al., 2004; Staroswiecki et al., 2009; 2012; Yu and Jiang, 2012).

In the last decades, the linear parameter varying (LPV) paradigm has become a standard formalism in systems and control, for analysis, controller synthesis and system identification (Shamma, 2012). This class of systems is important because, by embedding the system nonlinearities in the varying parameters, gain-scheduling control of nonlinear systems can be performed using an extension of linear techniques (in this case, the system is referred to as quasi-LPV since the varying parameters depend on exogenous signals). Some applications of LPV control theory to quadrotor UAVs can be found in the recent literature (Budiyono and Sutarto, 2006; Rangajeeva and Whidborne, 2011; Serirojanakul and Wongaisuwan, 2012; Rotondo et al., 2014).

Recently, the robust LPV polytopic framework, obtained by extending known results from the robust polytopic and the LPV polytopic control area has been introduced (Rotondo et al., 2013a; 2013c). In the proposed framework, the vector of varying parameters is used to schedule between uncertain LTI systems. The resulting approach consists in using a double-layer polytopic description to take into account both variability due to the parameter vector and uncertainty. The first polytopic layer manages the varying parameters and is used to obtain vertex uncertain systems, where vertex controllers are designed. The second polytopic layer is built in each vertex system to take into account model uncertainties and add robustness in the design step.

In this paper, a solution to the fault tolerant control problem is proposed for a quadrotor UAV. This solution relies on the use of a reference model that describes the desired trajectory. The idea of using a model reference-based control is well-established in the LTI framework (Landau, 1979) and has been recently extended to cope with the control of LPV systems (Abdullah and Zribi, 2009). Depending on the type of reference model used for generating the reference trajectory and on the assumptions about the availability and uncertainty of fault estimation, different error models are obtained. In the first one, faults enter into the system as if they were perturbations, making such an error model suitable for passive FTC (see Fig. 1). The second one is scheduled by faults, and it is referred to as the active FTC error model (see Fig. 2). Finally, in the third one, the error model is scheduled by the fault and considers the fault estimation uncertainty as a perturbation and an uncertainty at the same time: this model will be used for hybrid FTC (the scheme shown in Fig. 2 is valid in this case, too). The controller is obtained using theoretical results from the robust LPV polytopic framework and linear matrix inequalities (LMIs), in order to achieve regional pole placement and $\mathcal{H}_{\infty}$ norm bounding constraints. Simulation results are used to compare the different FTC strategies.

It is worth highlighting that, in the active and hybrid FTC cases, the overall scheme should include a module that provides fault estimation using some available measurements and the knowledge about the mathematical model of the system, as shown in Fig. 2 Furthermore, a fault detection and isolation (FDI) module could be added in order to reduce on-line the number of faults taken into consideration by the fault tolerant controller, allowing increasing the obtainable performance, as shown by Rotondo et al. (2013c). However, the fault detection, fault isolation and fault estimation problems, for which some recent solutions have been proposed (Zhang et al., 2013; Izadi et al., 2010; 2011; Rotondo et al., 2012; Zhaohui and Noura, 2013; Aguilar-Sierra et al., 2014; Cen et al., 2014), are not considered in this article. Indeed, the main goal of this work is to propose an FTC strategy that efficiently takes into account the information available from a fault estimator, independently of the fault estimation algorithm considered, and to show that it is possible to increase the FTC robustness using a hybrid passive/active FTC approach thanks to the robust LPV framework.

The paper is structured as follows. Section 2 introduces the dynamic model of the quadrotor, the reference and error models that are used for passive FTC, active FTC and hybrid FTC. Section 3 presents the robust LPV framework and the error feedback controller design 


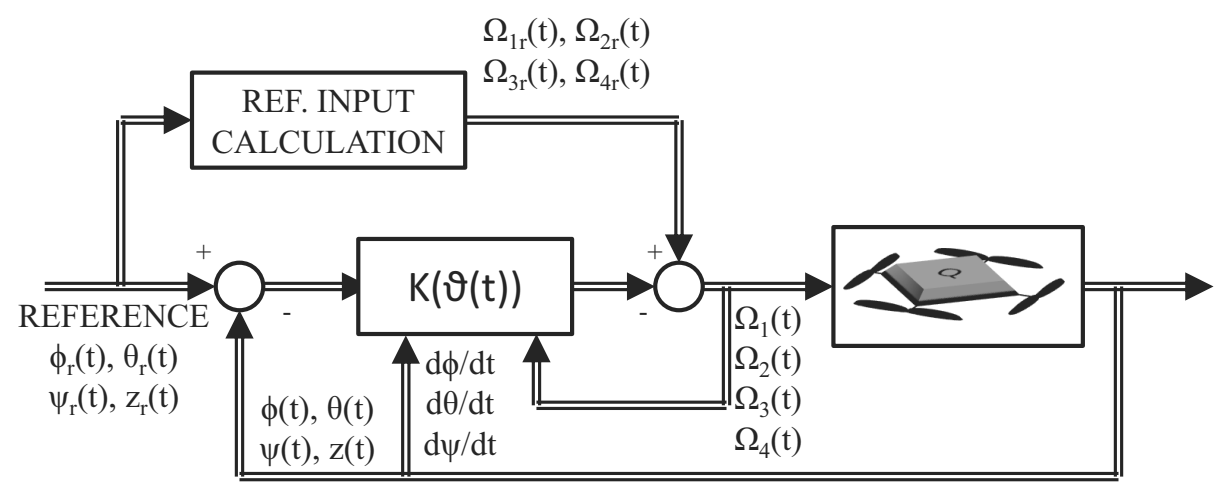

Fig. 1. Passive FTC scheme.

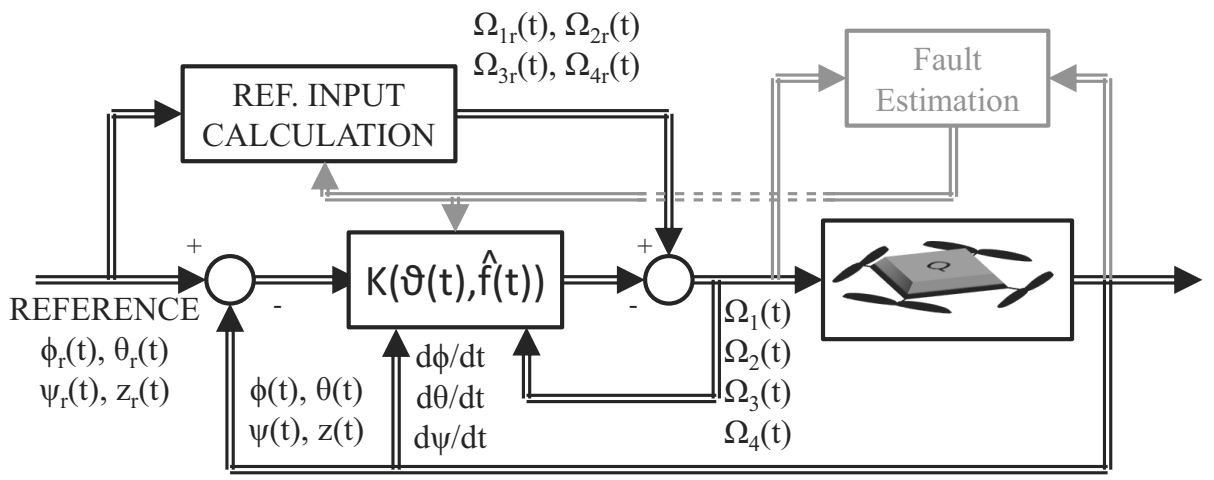

Fig. 2. Active and hybrid FTC schemes.

using LMI-based techniques. In Section 4, reference input calculation for trajectory tracking is discussed. Simulation results are shown in Section 5] Finally, the main conclusions are outlined in Section 6

\section{Quadrotor modeling}

The quadrotor is a vehicle that has four propellers in a cross configuration. Two propellers can rotate in a clockwise direction, while the other two can rotate anticlockwisely. The quadrotor is moved by changing the rotor speeds. For example, by increasing or decreasing together the four propeller speeds, a vertical motion is achieved. Changing only the speeds of the propellers situated oppositely produces either roll or lateral motions. Finally, a yaw rotation results from the difference in the counter-torque between each pair of propellers.

Let us consider an earth fixed frame $\{X Y Z\}$ and a body fixed frame $\left\{\begin{array}{lll}x_{b} & y_{b} & z_{b}\end{array}\right\}$ with the origin in the quadrotor center of mass. Under the assumptions that the body is rigid and symmetrical, and the propellers are rigid, i.e., no blade flapping occurs, the quadrotor faulty dynamic model is described by the following equations, obtained by Bouabdallah et al. (2004), adding multiplicative faults in the actuators $\left(\Omega_{i} \rightarrow f_{i} \Omega_{i}\right)$ :

$$
\begin{gathered}
\ddot{x}_{b}=(\cos \phi \sin \theta \cos \psi+\sin \phi \sin \psi) \frac{U_{1}^{f}}{m}, \\
\ddot{y}_{b}=(\cos \phi \sin \theta \sin \psi+\sin \phi \cos \psi) \frac{U_{1}^{f}}{m}, \\
\ddot{z}_{b}=-g+\cos \phi \cos \theta \frac{U_{1}^{f}}{m}, \\
\ddot{\phi}=\dot{\theta} \dot{\psi} \frac{I_{y}-I_{z}}{I_{x}}-\frac{J_{T P}}{I_{x}} \dot{\theta} \Omega_{f}+\frac{l U_{2}^{f}}{I_{x}}, \\
\ddot{\theta}=\dot{\phi} \dot{\psi} \frac{I_{z}-I_{x}}{I_{y}}+\frac{J_{T P}}{I_{y}} \dot{\phi} \Omega_{f}+\frac{l U_{3}^{f}}{I_{y}},
\end{gathered}
$$


Table 2. System parameters.

\begin{tabular}{|c|c|c|}
\hline Param. & Description & Value \\
\hline$\overline{I_{x}}$ & $\begin{array}{l}\text { Body moment of inertia } \\
\text { around the } x \text {-axis }\end{array}$ & $8.1 \times 10^{-3}\left[\mathrm{Nms}^{2}\right]$ \\
\hline$I_{y}$ & $\begin{array}{l}\text { Body moment of inertia } \\
\text { around the } y \text {-axis }\end{array}$ & $8.1 \times 10^{-3}\left[\mathrm{Nms}^{2}\right]$ \\
\hline$I_{z}$ & $\begin{array}{l}\text { Body moment of inertia } \\
\text { around the } z \text {-axis }\end{array}$ & $14.2 \times 10^{-3}\left[\mathrm{Nms}^{2}\right]$ \\
\hline$m$ & Mass of the quadrotor & $1[\mathrm{~kg}]$ \\
\hline$g$ & Acceleration due to gravity & $9.81\left[\mathrm{~ms}^{-2}\right]$ \\
\hline$J_{T P}$ & $\begin{array}{l}\text { Total rotational moment of } \\
\text { inertia around the propeller axis }\end{array}$ & $104 \times 10^{-6}\left[\mathrm{Nms}^{2}\right]$ \\
\hline$l$ & $\begin{array}{l}\text { Center of quadrotor to } \\
\text { center of propeller distance }\end{array}$ & $0.24[\mathrm{~m}]$ \\
\hline$b$ & Thrust factor & $54.2 \times 10^{-6}\left[\mathrm{Ns}^{2}\right]$ \\
\hline$d$ & Drag factor & $1.1 \times 10^{-6}\left[\mathrm{Nms}^{2}\right]$ \\
\hline
\end{tabular}

$$
\ddot{\psi}=\dot{\phi} \dot{\theta} \frac{I_{x}-I_{y}}{I_{z}}+\frac{U_{4}^{f}}{I_{z}},
$$

where $\phi$ is the roll angle, $\theta$ is the pitch angle, $\psi$ is the yaw angle, and the faulty inputs $U_{1}^{f}, U_{2}^{f}, U_{3}^{f}, U_{4}^{f}, \Omega_{f}$ are defined as follows:

$$
\begin{gathered}
U_{1}^{f}=b\left(f_{1}^{2} \Omega_{1}^{2}+f_{2}^{2} \Omega_{2}^{2}+f_{3}^{2} \Omega_{3}^{2}+f_{4}^{2} \Omega_{4}^{2}\right), \\
U_{2}^{f}=b\left(f_{4}^{2} \Omega_{4}^{2}-f_{2}^{2} \Omega_{2}^{2}\right), \\
U_{3}^{f}=b\left(f_{3}^{2} \Omega_{3}^{2}-f_{1}^{2} \Omega_{1}^{2}\right), \\
U_{4}^{f}=d\left(f_{2}^{2} \Omega_{2}^{2}+f_{4}^{2} \Omega_{4}^{2}-f_{1}^{2} \Omega_{1}^{2}-f_{3}^{2} \Omega_{3}^{2}\right), \\
\Omega_{f}=f_{2} \Omega_{2}+f_{4} \Omega_{4}-f_{1} \Omega_{1}-f_{3} \Omega_{3},
\end{gathered}
$$

where $f_{i}$ and $\Omega_{i}$ denote the $i$-th rotor fault magnitude and speed, respectively ( $f_{i}=1$ corresponds to the healthy rotor while $f_{i}=0$ corresponds to its total loss). For a description of the system parameters, as well as the values used in the simulations that are taken from Bresciani (2008); see Table2

In this paper, only the problem of attitude/altitude tracking control will be addressed. Hence, the dynamics of the system along the $x_{b}$ and $y_{b}$ axes, i.e., Eqns. (10) and (2), will be neglected.

2.1. Passive FTC reference model. In passive FTC, no information about the fault is available on-line. Hence, the same reference model used for the nominal case, and described by Rotondo et al. (2014), should be used as follows:

$$
\begin{gathered}
\dot{\phi}_{r}=v_{\phi}^{r}, \\
\dot{v}_{\phi}^{r}=\dot{\theta} v_{\psi}^{r} \frac{I_{y}-I_{z}}{2 I_{x}}+v_{\theta}^{r} \dot{\psi} \frac{I_{y}-I_{z}}{2 I_{x}}-\frac{J_{T P}}{I_{x}} \dot{\theta} \Omega_{r}+\frac{l U_{2}^{r}}{I_{x}}, \\
\dot{\theta}_{r}=v_{\theta}^{r}, \\
\dot{v}_{\theta}^{r}=\dot{\phi} v_{\psi}^{r} \frac{I_{z}-I_{x}}{2 I_{y}}+v_{\phi}^{r} \dot{\psi} \frac{I_{z}-I_{x}}{2 I_{y}}+\frac{J_{T P}}{I_{y}} \dot{\phi} \Omega_{r}+\frac{l U_{3}^{r}}{I_{y}},
\end{gathered}
$$

$$
\begin{gathered}
\dot{\psi}_{r}=v_{\psi}^{r}, \\
\dot{v}_{\psi}^{r}=v_{\phi}^{r} \dot{\theta} \frac{I_{x}-I_{y}}{2 I_{z}}+\dot{\phi} v_{\theta}^{r} \frac{I_{x}-I_{y}}{2 I_{z}}+\frac{d U_{4}^{r}}{I_{z}}, \\
\dot{z}_{r}=v_{z}^{r}, \\
\dot{v}_{z}^{r}=-g+\cos \phi \cos \theta \frac{U_{1}^{r}}{m},
\end{gathered}
$$

where $\phi_{r}$ is the reference roll angle, $\theta_{r}$ is the reference pitch angle, $\psi_{r}$ is the reference yaw angle, $z_{r}$ is the reference height, $v_{\phi}^{r}, v_{\theta}^{r}, v_{\psi}^{r}, v_{z}^{r}$ are the corresponding derivatives, and the reference inputs $U_{1}^{r}, U_{2}^{r}, U_{3}^{r}, U_{4}^{r}, \Omega_{r}$ are defined as follows:

$$
\begin{gathered}
U_{1}^{r}=b\left(\Omega_{1} \Omega_{1 r}+\Omega_{2} \Omega_{2 r}+\Omega_{3} \Omega_{3 r}+\Omega_{4} \Omega_{4 r}\right), \\
U_{2}^{r}=b\left(\Omega_{4} \Omega_{4 r}-\Omega_{2} \Omega_{2 r}\right), \\
U_{3}^{r}=b\left(\Omega_{3} \Omega_{3 r}-\Omega_{1} \Omega_{1 r}\right), \\
U_{4}^{r}=d\left(\Omega_{2} \Omega_{2 r}+\Omega_{4} \Omega_{4 r}-\Omega_{1} \Omega_{1 r}-\Omega_{3} \Omega_{3 r}\right), \\
\Omega_{r}=\Omega_{2 r}+\Omega_{4 r}-\Omega_{1 r}-\Omega_{3 r},
\end{gathered}
$$

where $\Omega_{i r}$ denotes the $i$-th reference rotor speed.

2.2. Active FTC reference model. In active FTC, an estimate of the faults, denoted in the following by $\hat{f}_{i}$, is available. This information is added to the reference model (12)-19) by changing $U_{1}^{r}, U_{2}^{r}, U_{3}^{r}, U_{4}^{r}, \Omega_{r}$ in (20)-(24) with the following values:

$$
\begin{gathered}
U_{1}^{r}=b\left(\hat{f}_{1}^{2} \Omega_{1} \Omega_{1 r}+\hat{f}_{2}^{2} \Omega_{2} \Omega_{2 r}\right. \\
\left.+\hat{f}_{3}^{2} \Omega_{3} \Omega_{3 r}+\hat{f}_{4}^{2} \Omega_{4} \Omega_{4 r}\right) \\
U_{2}^{r}=b\left(\hat{f}_{4}^{2} \Omega_{4} \Omega_{4 r}-\hat{f}_{2}^{2} \Omega_{2} \Omega_{2 r}\right), \\
U_{3}^{r}=b\left(\hat{f}_{3}^{2} \Omega_{3} \Omega_{3 r}-\hat{f}_{1}^{2} \Omega_{1} \Omega_{1 r}\right), \\
U_{4}^{r}=d\left(\hat{f}_{2}^{2} \Omega_{2} \Omega_{2 r}+\hat{f}_{4}^{2} \Omega_{4} \Omega_{4 r}-\hat{f}_{1}^{2} \Omega_{1} \Omega_{1 r}-\hat{f}_{3}^{2} \Omega_{3} \Omega_{3 r}\right), \\
\Omega_{r}=\hat{f}_{2} \Omega_{2 r}+\hat{f}_{4} \Omega_{4 r}-\hat{f}_{1} \Omega_{1 r}-\hat{f}_{3} \Omega_{3 r} .
\end{gathered}
$$

2.3. Passive FTC error model. By defining the tracking errors $e_{1} \triangleq \phi_{r}-\phi, e_{2} \triangleq v_{\phi}^{r}-\dot{\phi}, e_{3} \triangleq \theta_{r}-\theta$, $e_{4} \triangleq v_{\theta}^{r}-\dot{\theta}, e_{5} \triangleq \psi_{r}-\psi, e_{6} \triangleq v_{\psi}^{r}-\dot{\psi}, e_{7} \triangleq$ $z_{r}-z_{b}, e_{8} \triangleq v_{z}^{r}-\dot{z}_{b}$, the new inputs $o_{i} \triangleq \Omega_{i r}-\Omega_{i}$, $i=1,2,3,4$, and rewriting the faults as $\Delta f_{i}=f_{i}-1$, the error model for passive FTC of the quadrotor can be obtained from (3)-(24) and brought to a quasi-LPV representation following the non-linear embedding in the parameters approach proposed by Kwiatkowski et al. (2006) as follows:

$$
\begin{aligned}
\dot{e}(t)= & A(\vartheta(t)) e(t)+B(\vartheta(t)) o(t) \\
& +D(\vartheta(t)) \Delta f(t)
\end{aligned}
$$


where the vector of varying parameters is

$$
\vartheta(t)=\left[\begin{array}{c}
\vartheta_{1}(t) \\
\vartheta_{2}(t) \\
\vartheta_{3}(t) \\
\vartheta_{4}(t) \\
\vartheta_{5}(t) \\
\vartheta_{6}(t) \\
\vartheta_{7}(t) \\
\vartheta_{8}(t) \\
\vartheta_{9}(t) \\
\vartheta_{10}(t) \\
\vartheta_{11}(t) \\
\vartheta_{12}(t)
\end{array}\right]=\left[\begin{array}{c}
\dot{\phi}(t) \\
\dot{\theta}(t) \\
\dot{\psi}(t) \\
\Omega_{1}^{2}(t) \\
\Omega_{1}(t) \\
\Omega_{2}^{2}(t) \\
\Omega_{2}(t) \\
\Omega_{3}^{2}(t) \\
\Omega_{3}(t) \\
\Omega_{4}^{2}(t) \\
\Omega_{4}(t) \\
\cos \phi(t) \cos \theta(t)
\end{array}\right],
$$

and the matrices $A(\vartheta(t)), B(\vartheta(t))$ and $D(\vartheta(t))$ are defined by (32), (33) and (34).

2.4. Active FTC error model. The error model for active FTC of the quadrotor can be obtained from (3) - 19) and 25 -29) considering $f_{i}=\hat{f}_{i}, i=1,2,3,4$, and brought to a quasi-LPV representation as follows (Kwiatkowski et al., 2006):

$$
\dot{e}(t)=A(\vartheta(t)) e(t)+B(\vartheta(t)) o(t),
$$

where the vector of varying parameters is

$$
\vartheta(t)=\left[\begin{array}{c}
\vartheta_{1}(t) \\
\vartheta_{2}(t) \\
\vartheta_{3}(t) \\
\vartheta_{4}(t) \\
\vartheta_{5}(t) \\
\vartheta_{6}(t) \\
\vartheta_{7}(t) \\
\vartheta_{8}(t) \\
\vartheta_{9}(t) \\
\vartheta_{10}(t) \\
\vartheta_{11}(t) \\
\vartheta_{12}(t) \\
\vartheta_{13}(t) \\
\vartheta_{14}(t) \\
\vartheta_{15}(t) \\
\vartheta_{16}(t)
\end{array}\right]=\left[\begin{array}{c}
\dot{\phi}(t) \\
\dot{\theta}(t) \\
\dot{\psi}(t) \\
\Omega_{1}(t) \\
\Omega_{2}(t) \\
\Omega_{3}(t) \\
\Omega_{4}(t) \\
\hat{f}_{1}^{2}(t) \\
\hat{f}_{1}(t) \\
\hat{f}_{2}^{2}(t) \\
\hat{f}_{2}(t) \\
\hat{f}_{3}^{2}(t) \\
\hat{f}_{3}(t) \\
\hat{f}_{4}^{2}(t) \\
\hat{f}_{4}(t) \\
\cos \phi(t) \cos \theta(t)
\end{array}\right]
$$

the matrix $A(\vartheta(t))$ is defined as in (32), and the matrix $B(\vartheta(t))$ is defined by (37).

2.5. Hybrid FTC error model. Fault estimation algorithms are affected by uncertainties that will cause a difference between the fault estimated value, given by the algorithm, and the real fault value. Among the causes of uncertainty, there are the presence of external disturbances, the mismatch between the real and modeled dynamics, due to unmodeled nonlinearities and errors in the calibration of the model parameters during the identification phase, and the noise affecting the measurements given by the sensors. The presence of these uncertainties in fault estimation, if not properly taken into account, can degrade the fault tolerant control system performances and give rise to undesired behaviours. This fact motivates a combination of the benefits of passive and active FTC strategies in order to obtain a hybrid passive/active FTC.

The error model for hybrid passive/active FTC of the quadrotor can be obtained from (3) - 19) and (25)-29) considering $f_{i}=\hat{f}_{i}+\Delta f_{i}, i=1,2,3,4$. Then, the resulting quasi-LPV representation (Kwiatkowski et al., 2006) has the same structure of the passive FTC error model (30) with the vector of varying parameters made up by the one of active FTC error models 36 plus the following varying parameters:

$$
\left[\begin{array}{c}
\vartheta_{17}(t) \\
\vartheta_{18}(t) \\
\vartheta_{19}(t) \\
\vartheta_{20}(t)
\end{array}\right]=\left[\begin{array}{l}
\Omega_{1}^{2}(t) \\
\Omega_{2}^{2}(t) \\
\Omega_{3}^{2}(t) \\
\Omega_{4}^{2}(t)
\end{array}\right],
$$

where the matrix $A(\vartheta(t))$ is defined by (32), the matrix $B(\vartheta(t))$ is defined by (37), and the matrix $D(\vartheta(t))$ is defined by (39).

\section{Robust LPV framework}

In this paper, a framework based on a combination of robust and LPV polytopic designs is proposed. In this framework, the variation in the state matrix is due to the vector of varying parameters $\vartheta$, whose measurement or estimate is assumed to be available, together with some bounded uncertainties. The nominal LPV model is used to generate a polytope described by its vertices. Later, the model uncertainties are taken into account generating more polytopes, one for each vertex of the nominal polytope. The robust LPV polytopic design problem involves obtaining a controller scheduled by $\vartheta(t)$ as a combination of vertex controllers, each of which is designed to satisfy some LMI conditions at all vertices of the vertex polytope. Under some assumptions, the final result will be an LPV controller scheduled by $\vartheta$ that is robust against bounded uncertainties.

In particular, consider a continuous-time uncertain LPV system as in (30), where $e(t) \in \mathbb{R}^{n_{e}}$ is the state, $o(t) \in \mathbb{R}^{n_{o}}$ is the control input, $\Delta f(t) \in \mathbb{R}^{n_{f}}$ is a vector of exogenous inputs, $\vartheta(t) \in \Theta \subset \mathbb{R}^{n_{\vartheta}}$ is the vector of varying parameters and $A(\vartheta(t)), B$ (assumed to be constant), $D(\vartheta(t))$ are matrices of appropriate

\footnotetext{
${ }^{1}$ Notice that $\Delta f_{i}$ used in the passive FTC error model is different from $\Delta f_{i}$ employed in the hybrid FTC error model. However, the same notation is used because the design procedure described in this paper deals with both of them in the same way.
} 


$$
\begin{aligned}
& A(\cdot)=\left(\begin{array}{cccccccc}
0 & 1 & 0 & 0 & 0 & 0 & 0 & 0 \\
0 & 0 & 0 & \vartheta_{3} \frac{\left(I_{y}-I_{z}\right)}{2 I_{x}} & 0 & \vartheta_{2} \frac{\left(I_{y}-I_{z}\right)}{2 I_{x}} & 0 & 0 \\
0 & 0 & 0 & 1 & 0 & 0 & 0 & 0 \\
0 & \vartheta_{3} \frac{\left(I_{z}-I_{x}\right)}{2 I_{y}} & 0 & 0 & 0 & \vartheta_{1} \frac{\left(I_{z}-I_{x}\right)}{2 I_{y}} & 0 & 0 \\
0 & 0 & 0 & 0 & 0 & 1 & 0 & 0 \\
0 & \vartheta_{2} \frac{\left(I_{x}-I_{y}\right)}{2 I_{z}} & 0 & \vartheta_{1} \frac{\left(I_{x}-I_{y}\right)}{2 I_{z}} & 0 & 0 & 0 & 0 \\
0 & 0 & 0 & 0 & 0 & 0 & 0 & 1 \\
0 & 0 & 0 & 0 & 0 & 0 & 0 & 0
\end{array}\right), \\
& B(\cdot)=\left(\begin{array}{cccc}
0 & 0 & 0 & 0 \\
\frac{J_{T P}}{I_{x}} \vartheta_{2} & -\frac{J_{T P}}{I_{x}} \vartheta_{2}-\frac{l b}{I_{x}} \vartheta_{7} & \frac{J_{T P}}{I_{x}} \vartheta_{2} & -\frac{J_{T P}}{I_{x}} \vartheta_{2}+\frac{l b}{I_{x}} \vartheta_{11} \\
0 & 0 & 0 & 0 \\
-\frac{J_{T P}}{I_{y}} \vartheta_{1}-\frac{l b}{I_{y}} \vartheta_{5} & \frac{J_{T P}}{I_{y}} \vartheta_{1} & -\frac{J_{T P}}{I_{y}} \vartheta_{1}+\frac{l b}{I_{y}} \vartheta_{9} & \frac{J_{T P}}{I_{y}} \vartheta_{1} \\
0 & 0 & 0 & 0 \\
-\frac{d}{I_{z}} \vartheta_{5} & \frac{d}{I_{z}} \vartheta_{7} & -\frac{d}{I_{z}} \vartheta_{9} & \frac{d}{I_{z}} \vartheta_{11} \\
\frac{b}{0} \vartheta_{5} \vartheta_{12} & \frac{b}{m} \vartheta_{7} \vartheta_{12} & \frac{b}{m} \vartheta_{9} \vartheta_{12} & \frac{b}{m} \vartheta_{11} \vartheta_{12}
\end{array}\right), \\
& D(\cdot)=\left(\begin{array}{cc}
0 & 0 \\
-\frac{J_{T P}}{I_{x}} \vartheta_{5} & \frac{J_{T P}}{I_{x}} \vartheta_{7}+\frac{l b\left(2+\Delta f_{2}\right)}{I_{x}} \vartheta_{6} \\
0 & 0 \\
\frac{J_{T P}}{I_{y}} \vartheta_{5}+\frac{l b\left(2+\Delta f_{1}\right)}{I_{y}} \vartheta_{4} & -\frac{J_{T P}}{I_{y}} \vartheta_{7} \\
0 & 0 \\
\frac{d\left(2+\Delta f_{1}\right)}{I_{z}} \vartheta_{4} & -\frac{d\left(2+\Delta f_{2}\right)}{I_{z}} \vartheta_{6} \\
-\frac{b\left(2+\Delta f_{1}\right)}{m} \vartheta_{4} \vartheta_{12} & -\frac{b\left(2+\Delta f_{2}\right)}{m} \vartheta_{6} \vartheta_{12} \\
0 & 0
\end{array}\right. \\
& -\frac{J_{T P}}{I_{x}} \vartheta_{9} \quad \frac{J_{T P}}{I_{x}} \vartheta_{11}-\frac{l b\left(2+\Delta f_{4}\right)}{I_{x}} \vartheta_{10} \\
& \frac{J_{T P}}{I_{y}} \vartheta_{9}-\frac{l b\left(2+\Delta f_{3}\right)}{I_{y}} \vartheta_{8} \quad-\frac{J_{T P}}{I_{y}} \vartheta_{11} \\
& \frac{d\left(2+\Delta f_{3}\right)}{I_{z}} \vartheta_{8} \\
& -\frac{d\left(2+\Delta f_{4}\right)}{I_{z}} \vartheta_{10} \\
& -\frac{b\left(2+\Delta f_{3}\right)}{m} \vartheta_{8} \vartheta_{12} \quad-\frac{b\left(2+\Delta f_{4}\right)}{m} \vartheta_{10} \vartheta_{12}
\end{aligned}
$$




$$
B(\cdot)=\left(\begin{array}{cccc}
0 & 0 & 0 & 0 \\
\frac{J_{T P}}{I_{x}} \vartheta_{2} \vartheta_{9} & -\frac{J_{T P}}{I_{x}} \vartheta_{2} \vartheta_{11}-\frac{l b}{I_{x}} \vartheta_{5} \vartheta_{10} & \frac{J_{T P}}{I_{x}} \vartheta_{2} \vartheta_{13} & -\frac{J_{T P}}{I_{x}} \vartheta_{2} \vartheta_{15}+\frac{l b}{I_{x}} \vartheta_{7} \vartheta_{14} \\
0 & 0 & 0 & 0 \\
-\frac{J_{T P}}{I_{y}} \vartheta_{1} \vartheta_{9}-\frac{l b}{I_{y}} \vartheta_{4} \vartheta_{8} & \frac{J_{T P}}{I_{y}} \vartheta_{1} \vartheta_{11} & -\frac{J_{T P}}{I_{y}} \vartheta_{1} \vartheta_{13}+\frac{l b}{I_{y}} \vartheta_{6} \vartheta_{12} & \frac{J_{T P}}{I_{y}} \vartheta_{1} \vartheta_{15} \\
0 & \frac{d}{I_{z}} \vartheta_{5} \vartheta_{10} & 0 & \frac{d}{I_{z}} \vartheta_{7} \vartheta_{14} \\
-\frac{d}{I_{z}} \vartheta_{4} \vartheta_{8} & 0 & -\frac{d}{I_{z}} \vartheta_{6} \vartheta_{12} & 0 \\
\frac{b}{m} \vartheta_{16} \vartheta_{4} \vartheta_{8} & \frac{b}{m} \vartheta_{16} \vartheta_{5} \vartheta_{10} & \frac{b}{m} \vartheta_{16} \vartheta_{6} \vartheta_{12} & \frac{b}{m} \vartheta_{16} \vartheta_{7} \vartheta_{14}
\end{array}\right) .
$$

dimensions. Moreover, consider the additional algebraic equation

$$
h(t)=C(\vartheta(t)) e(t)+E o(t)+F(\vartheta(t)) \Delta f(t),
$$

where $h \in \mathbb{R}^{n_{h}}$ is a vector of output signals related to the $\mathcal{H}_{\infty}$ performance of the control system, and $C(\vartheta(t))$, $E, F(\vartheta(t))$ are matrices of appropriate dimensions. The system state-space matrices take values inside a polytope as follows:

$$
\left(\begin{array}{l}
A(\vartheta(t)) \\
C(\vartheta(t)) \\
D(\vartheta(t)) \\
F(\vartheta(t))
\end{array}\right)=\sum_{i=1}^{N} \alpha_{i}(\vartheta(t))\left(\begin{array}{c}
A_{i} \\
C_{i} \\
D_{i} \\
F_{i}
\end{array}\right)
$$

with

$$
\begin{aligned}
\sum_{i=1}^{N} \alpha_{i}(\vartheta(t))=1, \quad & \alpha_{i}(\vartheta(t)) \geq 0, \\
& \forall i=1, \ldots, N, \quad \vartheta \in \Theta .
\end{aligned}
$$

The matrices $A_{i}, C_{i}, D_{i}, F_{i}$ denote the vertices of $A(\vartheta(t)), C(\vartheta(t)), D(\vartheta(t)), F(\vartheta(t))$ at the $i$-th vertex of the polytope. Each of these matrices, together with $B$ and $E$, is uncertain, with an uncertainty that can be described as well in a polytopic way by $M$ LTI systems as follows:

$$
\left(\begin{array}{c}
A_{i} \\
B \\
C_{i} \\
D_{i} \\
E \\
F_{i}
\end{array}\right)=\sum_{j=1}^{M} \eta_{i j}\left(\begin{array}{c}
A_{i j} \\
B_{j} \\
C_{i j} \\
D_{i j} \\
E_{j} \\
F_{i j}
\end{array}\right)
$$

with

$$
\begin{aligned}
\sum_{j=1}^{M} \eta_{i j}=1, \quad \eta_{i j} & \geq 0, \\
i & =1, \ldots, N, \quad j=1, \ldots, M .
\end{aligned}
$$

The goal is to compute a polytopic LPV state-feedback control law:

$$
o(t)=K(\vartheta(t)) e(t)=\sum_{i=1}^{N} \alpha_{i}(\vartheta(t)) K_{i} e(t)
$$

that meets an $\mathcal{H}_{\infty}$ performance constraint and a regional pole 2 placement constraint on the closed-loop behaviour. These specifications must be satisfied in the robust LPV sense, that is, for each possible value that the parameter $\vartheta$ and the uncertain matrices $A, \ldots, F$ in (30) and (40) can take. In order to achieve this goal, the following theorem, namely, an extension of the results obtained by Chilali and Gahinet (1996), is used.

Theorem 1. Let $\mathcal{D}$ be an LMI region:

$$
\mathcal{D}=\left\{z \in \mathbb{C}: f_{\mathcal{D}}(z)<0\right\},
$$

with the characteristic function

$$
\begin{aligned}
f_{\mathcal{D}}(z) & =\alpha+z \beta+\bar{z} \beta^{T} \\
& =\left[\alpha_{k l}+\beta_{k l} z+\beta_{l k} \bar{z}\right]_{k, l \in[1, m]},
\end{aligned}
$$

and $\gamma>0$ being an upper bound on the closed-loop $\mathcal{H}_{\infty}$ performance. Assume that there exist a single Lyapunov matrix $X=X^{T}>0$ and $N$ matrices $\Gamma_{i}$ such that the following system of LMIs is feasible:

$$
\begin{gathered}
{\left[\alpha_{k l} X+\beta_{k l} U_{i j}\left(X, \Gamma_{i}\right)+\beta_{l k}\left(X, \Gamma_{i}^{T}\right)\right]_{k, l \in[1, m]}<0,} \\
\left(\begin{array}{ccc}
U_{i j}\left(X, \Gamma_{i}\right)+U_{i j}\left(X, \Gamma_{i}\right)^{T} & D_{i j} & V_{i j}\left(X, \Gamma_{i}\right)^{T} \\
D_{i j}^{T} & -I & F_{i j}^{T} \\
V_{i j}\left(X, \Gamma_{i}\right) & F_{i j} & -\gamma^{2} I
\end{array}\right)<0,
\end{gathered}
$$

with

$$
U_{i j}\left(X, \Gamma_{i}\right)=A_{i j}+B_{j} \Gamma_{i},
$$

\footnotetext{
${ }^{2}$ Notice that, following Ghersin and Sanchez-Peña (2002) and with a little abuse of language, the poles of an LPV system are defined as the set of all the poles of the LTI systems obtained by freezing $\vartheta(t)$ to all its possible values $\vartheta \in \Theta$.
} 


$$
\begin{aligned}
& \left(\begin{array}{cc}
0 & 0 \\
-\frac{J_{T P} \vartheta_{4}}{I_{x}} & \frac{J_{T P} \vartheta_{5}+l b\left(2 \hat{f}_{2}+\Delta f_{2}\right) \vartheta_{18}}{I_{x}} \\
& 0
\end{array}\right. \\
& D(\cdot)=\mid \begin{array}{cc}
\frac{J_{T P} \vartheta_{4}+l b\left(2 \hat{f}_{1}+\Delta f_{1}\right) \vartheta_{17}}{I_{y}} & -\frac{J_{T P} \vartheta_{18}}{I_{y}} \\
0 & 0 \\
\frac{d\left(2 \hat{f}_{1}+\Delta f_{1}\right) \vartheta_{17}}{I_{z}} & -\frac{d\left(2 \hat{f}_{2}+\Delta f_{2}\right) \vartheta_{18}}{I_{z}} \\
0 & 0
\end{array} \\
& -\frac{b\left(2 \hat{f}_{1}+\Delta f_{1}\right) \vartheta_{16} \vartheta_{17}}{m} \quad-\frac{b\left(2 \hat{f}_{2}+\Delta f_{2}\right) \vartheta_{16} \vartheta_{18}}{m} \\
& -\frac{J_{T P} \vartheta_{6}}{I_{x}} \\
& \frac{J_{T P} \vartheta_{6}}{I_{y}}-\frac{l b\left(2 \hat{f}_{3}+\Delta f_{3}\right) \vartheta_{19}}{I_{y}} \\
& \frac{J_{T P} \vartheta_{7}}{I_{x}}-\frac{l b\left(2 \hat{f}_{4}+\Delta f_{4}\right) \vartheta_{20}}{0} \\
& 0 \\
& -\frac{J_{T P} \vartheta_{7}}{I_{y}} \\
& d \frac{d\left(2 \hat{f}_{3}+\Delta f_{3}\right) \vartheta_{19}}{I_{z}} \\
& 0 \\
& -\frac{d\left(2 \hat{f}_{4}+\Delta f_{4}\right) \vartheta_{20}}{I_{z}} \\
& -\frac{b\left(2 \hat{f}_{3}+\Delta f_{3}\right) \vartheta_{16} \vartheta_{19}}{m} \\
& \left.-\frac{b\left(2 \hat{f}_{4}+\Delta f_{4}\right) \vartheta_{16} \vartheta_{20}}{m}\right)
\end{aligned}
$$

$$
V_{i j}\left(X, \Gamma_{i}\right)=C_{i j}+E_{j} \Gamma_{i} .
$$

Then, if $\left(X^{*}, \Gamma_{i}^{*}\right), i=1, \ldots, N$, is a solution of (48) and (49), the LPV state-feedback controller (45), with vertex gains calculated as $K_{i}=\Gamma_{i}^{*}\left(X^{*}\right)^{-1}$, satisfies the pole placement in $\mathcal{D}$ constraint and the $\mathcal{H}_{\infty}$ performance bound $\gamma$ in the robust LPV sense.

Proof. The pole placement in the $\mathcal{D}$ constraint and the $\mathcal{H}_{\infty}$ performance bound $\gamma$ are satisfied in the robust LPV sense if the following condition ${ }^{3}$ hold, $\forall \vartheta \in \Theta$ :

$$
\begin{gathered}
{\left[\alpha_{k l} X+\beta_{k l} U(X, \Gamma(\vartheta(t)))\right.} \\
\left.+\beta_{l k} U(X, \Gamma(\vartheta(t)))^{T}\right]_{k, l \in[1, m]}<0, \\
\left(\begin{array}{cc}
U(X, \Gamma(\vartheta(t)))+U(X, \Gamma(\vartheta(t)))^{T} \\
D(\vartheta(t))^{T} \\
V(X, \Gamma(\vartheta(t))) \\
D(\vartheta(t)) \quad V(X, \Gamma(\vartheta(t)))^{T} \\
-I \quad r(\vartheta(t))^{T} \\
F(\vartheta(t)) \quad-\gamma^{2} I
\end{array}\right)<0
\end{gathered}
$$

${ }^{3}$ These conditions are a consequence of the theorems presented by Chilali and Gahinet (1996). where

$$
\begin{aligned}
& U(X, \Gamma(\vartheta(t)))=A(\vartheta(t)) X+B \Gamma(\vartheta(t)), \\
& V(X, \Gamma(\vartheta(t)))=C(\vartheta(t)) X+E \Gamma(\vartheta(t)) .
\end{aligned}
$$

Taking into account (41)- 45, (52) can be rewritten as

$$
\begin{aligned}
{\left[\alpha_{k l} X\right.} & +\beta_{k l}\left(\sum_{i=1}^{N} \alpha_{i}(\vartheta(t)) \sum_{j=1}^{M} \eta_{i j} A_{i j} X\right. \\
& \left.+\sum_{j=1}^{M} \eta_{i j} B_{j} \sum_{i=1}^{N} \alpha_{i}(\vartheta(t)) \Gamma_{i}\right) \\
& +\beta_{l k}\left(\sum_{i=1}^{N} \alpha_{i}(\vartheta(t)) \sum_{j=1}^{M} \eta_{i j} X A_{i j}^{T}\right. \\
& \left.\left.+\sum_{i=1}^{N} \alpha_{i}(\vartheta(t)) \Gamma_{i}^{T} \sum_{j=1}^{M} \eta_{i j} B_{j}^{T}\right)\right]_{k, l \in[1, m]}<0
\end{aligned}
$$

which can be rewritten as

$$
\sum_{i=1}^{N} \alpha_{i}(\vartheta(t)) \sum_{j=1}^{M} \eta_{i j} \Phi_{i j}^{\mathcal{D}}<0
$$


with

$$
\begin{aligned}
\Phi_{i j}^{\mathcal{D}}= & {\left[\alpha_{k l} X+\beta_{k l}\left(A_{i j} X+B_{j} \Gamma_{i}\right)\right.} \\
& \left.+\beta_{l k}\left(A_{i j} X+B_{j} \Gamma_{i}\right)^{T}\right]_{k, l \in[1, m]}
\end{aligned}
$$

Similarly, (53) can be brought to the following form:

$$
\sum_{i=1}^{N} \alpha_{i}(\vartheta(t)) \sum_{j=1}^{M} \eta_{i j} \Phi_{i j}^{\infty}<0
$$

with

$$
\begin{aligned}
\Phi_{i j}^{\infty}= & \left(\begin{array}{c}
U_{i j}\left(X, \Gamma_{i}\right)+U_{i j}\left(X, \Gamma_{i}\right)^{T} \\
D_{i j}^{T} \\
V_{i j}\left(X, \Gamma_{i}\right)
\end{array}\right. \\
& \left.\begin{array}{cc}
D_{i j} & V_{i j}\left(X, \Gamma_{i}\right)^{T} \\
-I & F_{i j}^{T} \\
F_{i j} & -\gamma^{2} I
\end{array}\right)
\end{aligned}
$$

From the basic property of matrices (Horn and Johnson, 1990) that any linear combination of positive (resp. negative) definite matrices with non-negative coefficients, whose sum is positive, is positive (resp. negative) definite, (48) and (49) are obtained, and this completes the proof.

Notice that the hypothesis of fixed matrices $B$ and $E$ has been done. In many cases, this is not true and a prefiltering of the input $o(t)$ is needed in order to obtain a new system with constant matrices $\tilde{B}$ and $\tilde{E}$, as proposed by Apkarian et al. (1995). More specifically, defining a new control input $\tilde{o}(t)$ such that

$$
\begin{gathered}
\dot{x}_{o}(t)=A_{o}(\vartheta(t)) x_{o}(t)+B_{o} \tilde{o}(t), \\
o(t)=C_{o} x_{o}(t),
\end{gathered}
$$

with $A_{o}(\vartheta(t))$ being stable, the resulting LPV plant is described by

$$
\begin{gathered}
\left(\begin{array}{c}
\dot{e}(t) \\
\dot{x}_{o}(t)
\end{array}\right) \\
=\left(\begin{array}{cc}
A(\vartheta(t)) & B(\vartheta(t)) C_{o} \\
0 & A_{o}
\end{array}\right)\left(\begin{array}{c}
e(t) \\
x_{o}(t)
\end{array}\right) \\
+\left(\begin{array}{c}
0 \\
B_{o}
\end{array}\right) \tilde{o}(t)+\left(\begin{array}{c}
D(\vartheta(t)) \\
0
\end{array}\right) \Delta f(t), \\
h(t)=\left(\begin{array}{ll}
C(\vartheta(t)) & E(\vartheta(t)) C_{o}
\end{array}\right)\left(\begin{array}{c}
e(t) \\
x_{o}(t)
\end{array}\right) \\
+F(\vartheta(t)) \Delta f(t) .
\end{gathered}
$$

\section{Reference inputs calculation for trajectory tracking}

To make the quadrotor track a desired trajectory, proper values of $\Omega_{1 r}, \Omega_{2 r}, \Omega_{3 r}, \Omega_{4 r}$ should be fed to the reference model, such that its state equals the one corresponding to the desired trajectory.

Here, for illustrative purposes, the case of sinusoidal trajectories is considered as follows

$$
\begin{aligned}
& \phi_{r}(t)=\Phi \sin \left(\frac{2 \pi t}{N_{\phi}}\right), \\
& \theta_{r}(t)=\Theta \sin \left(\frac{2 \pi t}{N_{\theta}}\right), \\
& \psi_{r}(t)=\Psi \sin \left(\frac{2 \pi t}{N_{\psi}}\right), \\
& z_{r}(t)=Z \sin \left(\frac{2 \pi t}{N_{z}}\right),
\end{aligned}
$$

where $\Phi, \Theta, \Psi, Z$ are the amplitudes, and $N_{\phi}, N_{\theta}, N_{\psi}$, $N_{z}$ are the periods. Taking the derivatives of 65 -68 and considering (12), (14), (16), (18), respectively, the following is obtained:

$$
\begin{aligned}
& \dot{\phi}_{r}(t)=v_{\phi}^{r}(t)=\Phi \cos \left(\frac{2 \pi t}{N_{\phi}}\right) \frac{2 \pi}{N_{\phi}}, \\
& \dot{\theta}_{r}(t)=v_{\theta}^{r}(t)=\Theta \cos \left(\frac{2 \pi t}{N_{\theta}}\right) \frac{2 \pi}{N_{\theta}}, \\
& \dot{\psi}_{r}(t)=v_{\psi}^{r}(t)=\Psi \cos \left(\frac{2 \pi t}{N_{\psi}}\right) \frac{2 \pi}{N_{\psi}}, \\
& \dot{z}_{r}(t)=v_{z}^{r}(t)=Z \cos \left(\frac{2 \pi t}{N_{z}}\right) \frac{2 \pi}{N_{z}} .
\end{aligned}
$$

Then, another differentiation of (69)-72) leads to

$$
\begin{aligned}
& \ddot{\phi}_{r}(t)=\dot{v}_{\phi}^{r}(t)=-\Phi\left(\frac{2 \pi}{N_{\phi}}\right)^{2} \sin \left(\frac{2 \pi t}{N_{\phi}}\right), \\
& \ddot{\theta}_{r}(t)=\dot{v}_{\theta}^{r}(t)=-\Theta\left(\frac{2 \pi}{N_{\theta}}\right)^{2} \sin \left(\frac{2 \pi t}{N_{\theta}}\right), \\
& \ddot{\psi}_{r}(t)=\dot{v}_{\psi}^{r}(t)=-\Psi\left(\frac{2 \pi}{N_{\psi}}\right)^{2} \sin \left(\frac{2 \pi t}{N_{\psi}}\right), \\
& \ddot{z}_{r}(t)=\dot{v}_{z}^{r}(t)=-Z\left(\frac{2 \pi}{N_{z}}\right)^{2} \sin \left(\frac{2 \pi t}{N_{z}}\right),
\end{aligned}
$$

and, by properly replacing (69-176) into 13, 15, 17 and (19), and taking into account (25)-29), we obtain

$$
\begin{aligned}
\dot{\theta} \Psi \cos \left(\frac{2 \pi t}{N_{\psi}}\right) \frac{2 \pi}{N_{\psi}} \frac{I_{y}-I_{z}}{2 I_{x}} \\
+\dot{\psi} \Theta \cos \left(\frac{2 \pi t}{N_{\theta}}\right) \frac{2 \pi}{N_{\theta}} \frac{I_{y}-I_{z}}{2 I_{x}} \\
\quad-\frac{J_{T P}}{I_{x}} \dot{\theta}\left(\hat{f}_{2} \Omega_{2 r}+\hat{f}_{4} \Omega_{4 r}-\hat{f}_{1} \Omega_{1 r}-\hat{f}_{3} \Omega_{3 r}\right) \\
+\frac{l b}{I_{x}}\left[\hat{f}_{4}^{2}\left(\Omega_{4 r}-o_{4}\right) \Omega_{4 r}-\hat{f}_{2}^{2}\left(\Omega_{2 r}-o_{2}\right) \Omega_{2 r}\right] \\
+\Phi\left(\frac{2 \pi}{N_{\phi}}\right)^{2} \sin \left(\frac{2 \pi t}{N_{\phi}}\right)=0,
\end{aligned}
$$




$$
\begin{aligned}
\dot{\phi} \Psi & \cos \left(\frac{2 \pi t}{N_{\psi}}\right) \frac{2 \pi}{N_{\psi}} \frac{I_{z}-I_{x}}{2 I_{y}} \\
& +\dot{\psi} \Phi \cos \left(\frac{2 \pi t}{N_{\phi}}\right) \frac{2 \pi}{N_{\phi}} \frac{I_{z}-I_{x}}{2 I_{y}} \\
& +\frac{J_{T P}}{I_{y}} \dot{\phi}\left(\hat{f}_{2} \Omega_{2 r}+\hat{f}_{4} \Omega_{4 r}-\hat{f}_{1} \Omega_{1 r}-\hat{f}_{3} \Omega_{3 r}\right) \\
& +\frac{l b}{I_{y}}\left[\hat{f}_{3}^{2}\left(\Omega_{3 r}-o_{3}\right) \Omega_{3 r}-\hat{f}_{1}^{2}\left(\Omega_{1 r}-o_{1}\right) \Omega_{1 r}\right] \\
& +\Theta\left(\frac{2 \pi}{N_{\theta}}\right)^{2} \sin \left(\frac{2 \pi t}{N_{\theta}}\right)=0,
\end{aligned}
$$

$$
\begin{aligned}
\dot{\theta} \Phi & \cos \left(\frac{2 \pi t}{N_{\phi}}\right) \frac{2 \pi}{N_{\phi}} \frac{I_{x}-I_{y}}{2 I_{z}} \\
& +\dot{\phi} \Theta \cos \left(\frac{2 \pi t}{N_{\theta}}\right) \frac{2 \pi}{N_{\theta}} \frac{I_{x}-I_{y}}{2 I_{z}} \\
& +\frac{d}{I_{z}}\left[\hat{f}_{2}^{2}\left(\Omega_{2 r}-o_{2}\right) \Omega_{2 r}+\hat{f}_{4}^{2}\left(\Omega_{4 r}-o_{4}\right) \Omega_{4 r}\right] \\
& -\frac{d}{I_{z}}\left[\hat{f}_{1}^{2}\left(\Omega_{1 r}-o_{1}\right) \Omega_{1 r}+\hat{f}_{3}^{2}\left(\Omega_{3 r}-o_{3}\right) \Omega_{3 r}\right] \\
& +\Psi\left(\frac{2 \pi}{N_{\psi}}\right)^{2} \sin \left(\frac{2 \pi t}{N_{\psi}}\right)=0,
\end{aligned}
$$

$$
\begin{aligned}
& \frac{b \cos \phi \cos \theta}{m}\left[\hat{f}_{1}^{2}\left(\Omega_{1 r}-o_{1}\right) \Omega_{1 r}+\hat{f}_{2}^{2}\left(\Omega_{2 r}-o_{2}\right) \Omega_{2 r}\right] \\
& +\frac{b \cos \phi \cos \theta}{m}\left[\hat{f}_{3}^{2}\left(\Omega_{3 r}-o_{3}\right) \Omega_{3 r}+\hat{f}_{4}^{2}\left(\Omega_{4 r}-o_{4}\right) \Omega_{4 r}\right] \\
& -g+Z\left(\frac{2 \pi}{N_{z}}\right)^{2} \sin \left(\frac{2 \pi t}{N_{z}}\right)=0 .
\end{aligned}
$$

Given $\dot{\phi}, \dot{\theta}, \dot{\psi}$ (measured), $\hat{f}_{1}, \hat{f}_{2}, \hat{f}_{3}, \hat{f}_{4}$ (estimated), and $o_{1}, o_{2}, o_{3}, o_{4}$ (obtained using (45)), we get (77)- (80) as a system of nonlinear equations with unknown variables $\Omega_{1 r}, \Omega_{2 r}, \Omega_{3 r}, \Omega_{4 r}$ that can be solved using some appropriate solver, e.g., fsolve in the Matlab Optimization Toolbox (Coleman et al., 1999).

Notice that when the passive FTC reference model is considered, i.e., (20)-(24), the changes $\hat{f}_{i} \rightarrow 1, i=$ $1,2,3,4$ should be introduced in (77)-(80).

\section{Results}

The results presented in this section compare the proposed FTC strategies. As already discussed in Section 3, since the input matrix $B$ is not constant, a prefiltering of the inputs is needed to obtain a constant input matrix $\tilde{B}$. Adding the states $x_{O_{1}}, x_{O_{2}}, x_{O_{3}}$ and $x_{O_{4}}$ to the error vector such that $o_{i}(t)=x_{o_{i}}(t)$, this corresponds to the case $C_{o}=I$ in (62), with the state equation (61) given by

$$
\dot{x}_{o_{i}}(t)=-\omega_{i} x_{o_{i}}(t)+\omega_{i} \tilde{o}_{i}(t),
$$

where $\tilde{o}_{i}(t), i=1, \ldots, 4$ are the new inputs, and $\omega_{i}$ has been chosen as $\omega_{i}=100, i=1, \ldots, 4$.

The polytopic approximation (41) of the quadrotor quasi-LPV passive FTC error model (30)-(34) was obtained by considering

$$
\begin{gathered}
\vartheta_{1} \in[\min (\dot{\phi}), \max (\dot{\phi})]=[-0.25,0.25], \\
\vartheta_{2} \in[\min (\dot{\theta}), \max (\dot{\theta})]=[-0.25,0.25], \\
\vartheta_{3} \in[\min (\dot{\psi}), \max (\dot{\psi})]=[-0.25,0.25], \\
\left(\begin{array}{c}
\vartheta_{2 i+2} \\
\vartheta_{2 i+3}
\end{array}\right) \in \operatorname{Tr}\left\{\begin{array}{c}
\min \left(\Omega_{i}\right)^{2} \\
\min \left(\Omega_{i}\right)
\end{array}\right),\left(\begin{array}{c}
\min \left(\Omega_{i}\right)^{2} \\
\max \left(\Omega_{i}\right)
\end{array}\right), \\
\left.\left.\quad \begin{array}{c}
\max \left(\Omega_{i}\right)^{2} \\
\max \left(\Omega_{i}\right)
\end{array}\right)\right\},
\end{gathered}
$$

with $\min \left(\Omega_{i}\right)=100, \max \left(\Omega_{i}\right)=500, i=1,2,3,4$, and where ' $\mathrm{Tr}$ ' denotes a triangular polytopic approximation, which was preferred to a bounding box one in order to reduce the conservativeness. Finally, $\vartheta_{8} \in[0.5,1]$, which corresponds to the interval of possible values of $\vartheta_{8}$ when $\phi \in[-\pi / 4, \pi / 4]$ and $\theta \in[-\pi / 4, \pi / 4]$.

The polytopic approximation (41) of the quadrotor quasi-LPV active FTC error model (35)-(37) was obtained by considering

$$
\begin{gathered}
\vartheta_{1} \in[\min (\dot{\phi}), \max (\dot{\phi})]=[-0.25,0.25], \\
\vartheta_{2} \in[\min (\dot{\theta}), \max (\dot{\theta})]=[-0.25,0.25], \\
\vartheta_{3} \in[\min (\dot{\psi}), \max (\dot{\psi})]=[-0.25,0.25], \\
\vartheta_{i+3} \in\left[\min \left(\Omega_{i}\right), \max \left(\Omega_{i}\right)\right]=[100,500], \\
i=1,2,3,4, \\
\left(\begin{array}{c}
\vartheta_{2 i+6} \\
\vartheta_{2 i+7}
\end{array}\right) \\
\in \operatorname{Tr}\left\{\left(\begin{array}{c}
\min \left(f_{i}\right)^{2} \\
\min \left(f_{i}\right)
\end{array}\right),\left(\begin{array}{c}
\min \left(f_{i}\right)^{2} \\
1
\end{array}\right),\left(\begin{array}{c}
1 \\
1
\end{array}\right)\right\},
\end{gathered}
$$

$$
\vartheta_{16} \in[0.5,1] .
$$

Similar considerations were applied to the quadrotor quasi-LPV hybrid FTC error model for obtaining its polytopic approximation. In particular, the results presented hereafter were obtained considering $\min \left(f_{i}\right)=$ 0.7 .

The passive/active/hybrid controllers were designed using (48)-(49), to assure stability and pole clustering in:

$$
\begin{aligned}
\mathcal{D}=\{z \in \mathbb{C}: & \operatorname{Re}(z)<-0.5, \operatorname{Re}(z)^{2}+\operatorname{Im}(z)^{2} \\
& <10000, \tan (0.3) \operatorname{Re}(z)<-|\operatorname{Im}(z)|\}
\end{aligned}
$$


and an $\mathcal{H}_{\infty}$ performance bound $\gamma=1000$, and considering $h(t)=\left[\begin{array}{llll}\phi & \theta & \psi & z\end{array}\right]^{T}$ in (40).

It must be remarked that, due to the exponential growth of the vertices with the number of faults taken into consideration $\left(2^{8} \times 3^{i}\right.$ vertices in the passive and active FTC cases, $2^{8-i} \times 3^{2 i}$ in the hybrid FTC case, where $i$ is the number of considered faults), the time needed to solve the LMIs grows exponentially, too. However, the strong calculating capacity available nowadays and the fact that the controller design is performed off-line and only the coefficients of the polytopic decomposition must be calculated on-line make this issue less critical.

The results shown in this paper refer to simulations which last $30 \mathrm{~s}$, where the quadrotor is driven from the initial state:

$$
\begin{aligned}
& \phi(0)=\frac{\pi}{6}, \quad \theta(0)=\frac{\pi}{6}, \quad \psi(0)=\frac{\pi}{6}, \quad z(0)=0, \\
& \dot{\phi}(0)=0, \quad \dot{\theta}(0)=0, \quad \dot{\psi}(0)=0, \quad \dot{z}(0)=0
\end{aligned}
$$

to the desired trajectory defined as in (77) -80 , with $\Phi=$ $\Theta=\Psi=0.1, Z=0, N_{\phi}=N_{\theta}=N_{\psi}=N_{z}=10 \mathrm{~s}$. The desired trajectory was generated by the reference model (12)-(19) starting from the initial reference state:

$$
\begin{aligned}
& \left(\phi_{r}(0), v_{\phi}^{r}(0), \theta_{r}(0), v_{\theta}^{r}(0), \psi_{r}(0), v_{\psi}^{r}(0), z_{r}(0), v_{z}^{r}(0)\right)^{T} \\
& =\left(0,2 \pi \Phi / N_{\phi}, 0,2 \pi \Theta / N_{\theta}, 0,2 \pi \Psi / N_{\psi}, 0,2 \pi Z / N_{z}\right)^{T}
\end{aligned}
$$

Figures 3,6 present a comparison between the responses obtained with a nominal controller and the ones obtained with the proposed passive FTC approach. A fault in the first actuator acts starting from the time instant $t=15 \mathrm{~s}$. It can be seen that even a small fault, e.g., $f_{1}=0.9$, is enough to drive the system to instability if the nominal controller is used. On the other hand, passive FTC shows some tolerance capability since, for $f_{1}=0.8$ and $f_{1}=0.9$, the stability is preserved, although with a steady-state error due to the effect of the faul 4 .

On the other hand, the proposed active FTC technique can achieve perfect fault tolerance as long as the fault is correctly estimated, as shown in Figs. $7+10$ (black solid line), where a fault $f_{1}=0.7$ acting from $t=15 \mathrm{~s}$ is considered. However, as the uncertainty in fault estimation (in this work modeled as a uniformly bounded noise) increases, so does the error between the real trajectory and the reference one.

By applying the proposed hybrid FTC method, the overall performance can be improved, thus reducing the effect that the fault estimation error has on the closed-loop response, as shown in Figs. 11,14

In order to quantify numerically the improvement brought by the FTC strategies considered, let us introduce

\footnotetext{
${ }^{4}$ Adding an integral action to the controller could eliminate the steady-state error, although at the expense of worsening the dynamical transient performance of the closed-loop system.
}

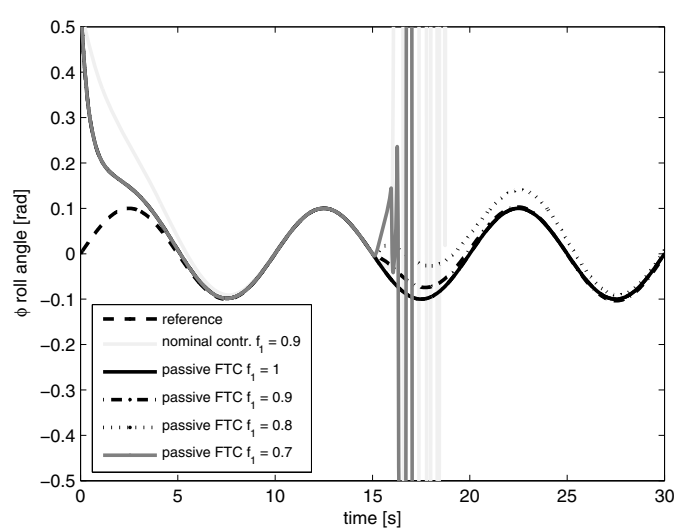

Fig. 3. Roll angle response (comparison between the nominal controller and passive FTC).

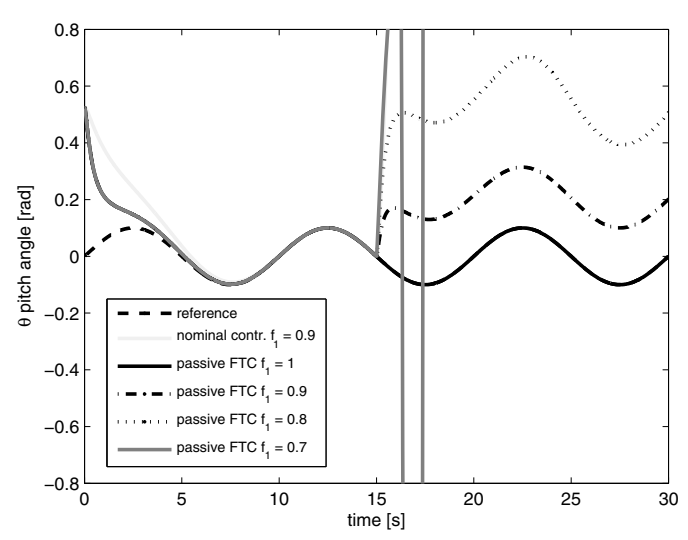

Fig. 4. Pitch angle response (comparison between the nominal controller and passive FTC).

the following performance measures:

$$
\begin{aligned}
& J_{\phi}=\frac{1}{1500} \sum_{k=1500}^{3000}\left(\phi_{r}\left(\frac{k}{100}\right)-\phi\left(\frac{k}{100}\right)\right)^{2}, \\
& J_{\theta}=\frac{1}{1500} \sum_{k=1500}^{3000}\left(\theta_{r}\left(\frac{k}{100}\right)-\theta\left(\frac{k}{100}\right)\right)^{2}, \\
& J_{\psi}=\frac{1}{1500} \sum_{k=1500}^{3000}\left(\psi_{r}\left(\frac{k}{100}\right)-\psi\left(\frac{k}{100}\right)\right)^{2}, \\
& J_{z}=\frac{1}{1500} \sum_{k=1500}^{3000}\left(z_{r}\left(\frac{k}{100}\right)-z\left(\frac{k}{100}\right)\right)^{2}, \\
& J=J_{\phi}+J_{\theta}+J_{\psi}+J_{z} .
\end{aligned}
$$

A comparison of the performance measures obtained in the different cases, as given in Table 3, shows the improvement brought by the proposed FTC strategies with respect to the nominal one, as well as the one brought by hybrid FTC with respect to the passive and active FTC strategies. 
Table 3. Comparison of nominal controllers with passive/active/hybrid FTC.

\begin{tabular}{|c|c|c|c|c|c|c|}
\hline $\begin{array}{c}\text { Type of FTC } \\
\text { strategy }\end{array}$ & $\begin{array}{c}\text { Fault/uncertainty } \\
\text { magnitude }\end{array}$ & $J_{\phi}$ & $J_{\theta}$ & $J_{\psi}$ & $J_{z}$ & $J$ \\
\hline \hline Nominal & $f_{1}=1$ & $3.90 \cdot 10^{-9}$ & $3.45 \cdot 10^{-9}$ & $4.12 \cdot 10^{-9}$ & $1.62 \cdot 10^{-11}$ & $1.15 \cdot 10^{-8}$ \\
& $f_{1}=0.9$ & 105.16 & 593.65 & 388.47 & $1.60 \cdot 10^{3}$ & $2.69 \cdot 10^{3}$ \\
Passive & $f_{1}=1$ & $1.39 \cdot 10^{-10}$ & $1.38 \cdot 10^{-10}$ & $1.19 \cdot 10^{-10}$ & $2.71 \cdot 10^{-10}$ & $6.67 \cdot 10^{-10}$ \\
& $f_{1}=0.9$ & $2.00 \cdot 10^{-4}$ & 0.0471 & 0.0114 & 0.0266 & 0.0853 \\
& $f_{1}=0.8$ & 0.0023 & 0.3246 & 0.0814 & 0.1400 & 0.5483 \\
Active & $f_{1}=0.7$ & $6.60 \cdot 10^{3}$ & $3.74 \cdot 10^{3}$ & $9.32 \cdot 10^{4}$ & $2.03 \cdot 10^{5}$ & $3.06 \cdot 10^{5}$ \\
& $f_{1}=0.7$ & & & & & \\
& $\Delta f_{1}=0$ & $5.73 \cdot 10^{-10}$ & $1.18 \cdot 10^{-8}$ & $9.36 \cdot 10^{-10}$ & $7.04 \cdot 10^{-11}$ & $1.34 \cdot 10^{-8}$ \\
& $\Delta f_{1} \in[-.01, .01]$ & $8.56 \cdot 10^{-8}$ & $1.77 \cdot 10^{-4}$ & $5.29 \cdot 10^{-7}$ & $7.21 \cdot 10^{-8}$ & $1.78 \cdot 10^{-4}$ \\
& $\Delta f_{1} \in[-.05, .05]$ & $2.37 \cdot 10^{-6}$ & 0.0056 & $1.69 \cdot 10^{-5}$ & $2.26 \cdot 10^{-6}$ & 0.0056 \\
& $\Delta f_{1} \in[-.10, .10]$ & $5.52 \cdot 10^{-6}$ & 0.0246 & $7.30 \cdot 10^{-5}$ & $1.02 \cdot 10^{-5}$ & 0.0247 \\
Hybrid & $f_{1}=0.7$ & $8.71 \cdot 10^{-6}$ & $3.91 \cdot 10^{-4}$ & $5.75 \cdot 10^{-5}$ & $1.25 \cdot 10^{-4}$ & $5.82 \cdot 10^{-4}$ \\
\hline
\end{tabular}

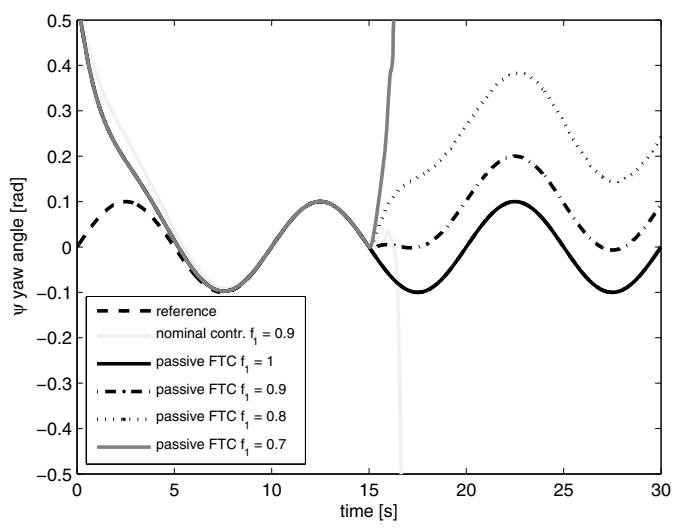

Fig. 5. Yaw angle response (comparison between the nominal controller and passive FTC).

\section{Conclusions}

In this paper, a solution for FTC of a quadrotor UAV has been proposed. By defining two reference models, different error models suitable for FTC can be obtained. In particular, three kinds of strategies can be used: (i) passive FTC, where faults are dealt with as though as they were exogenous perturbations, (ii) active FTC, where the controller is scheduled by the fault estimation, and (iii) $h y$ brid FTC, which combines the characteristics of passive and active FTC.

Controller design is performed within the robust LPV framework, where an LPV controller is designed to be scheduled by the vector of varying parameters and to be robust against bounded uncertainties, satisfying some conditions expressed as LMIs.

The results presented in the paper have shown the relevant features of the proposed FTC strategy, which is able to improve the performances under fault occurrence. In particular, whereas the passive FTC shows some limited tolerance capability, resulting in the appearance of steady-state errors due to the fault effect, the active

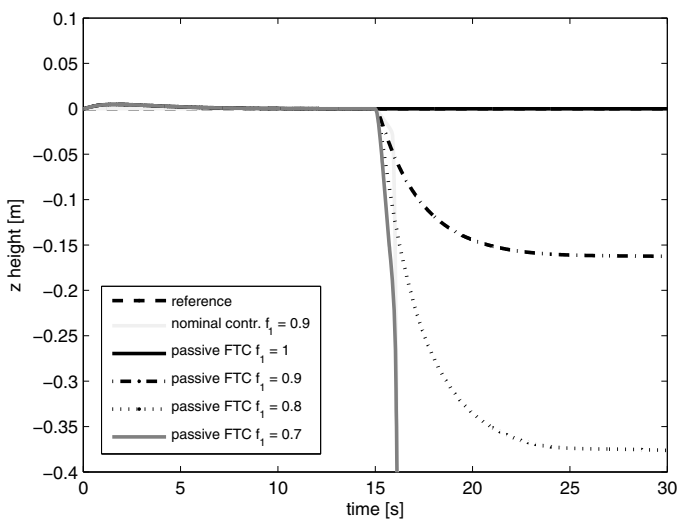

Fig. 6. Height response (comparison between the nominal controller and passive FTC).

FTC technique can achieve perfect fault tolerance as long as the fault is correctly estimated. However, as the uncertainty in fault estimation increases, so does the error between the real trajectory and the reference one. By applying the proposed hybrid FTC method, the overall performance can be improved, thus reducing the effect that the fault estimation error has on the closed-loop response. The introduction and comparison of some performance measures have allowed to numerically confirm such analysis.

Future research will be aimed at applying the proposed FTC strategy to a real set-up. This goal brings additional challenges, due to the presence of many sources of uncertainties that must be taken into account in order to enforce the robustness of the FTC strategy. Moreover, as remarked in the introduction, the inclusion of an FDI module can allow increasing the obtainable performance. Thus, further research will investigate FDI (as well as fault estimation) algorithms that can be successfully applied to quadrotor UAVs. 


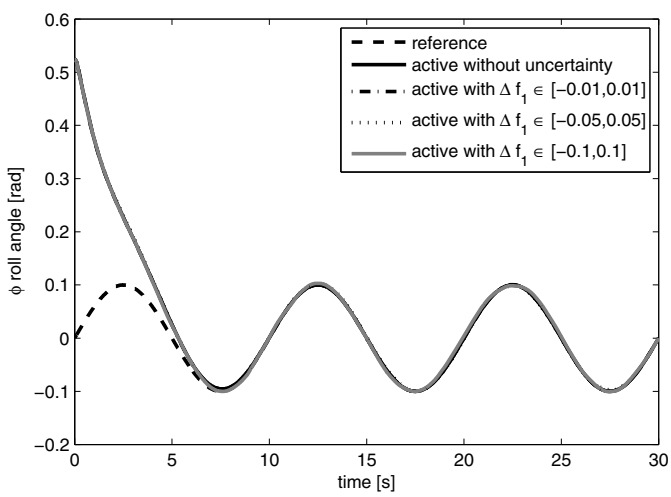

Fig. 7. Roll angle response (active FTC without and with uncertainty, $\left.f_{1}=0.7\right)$.

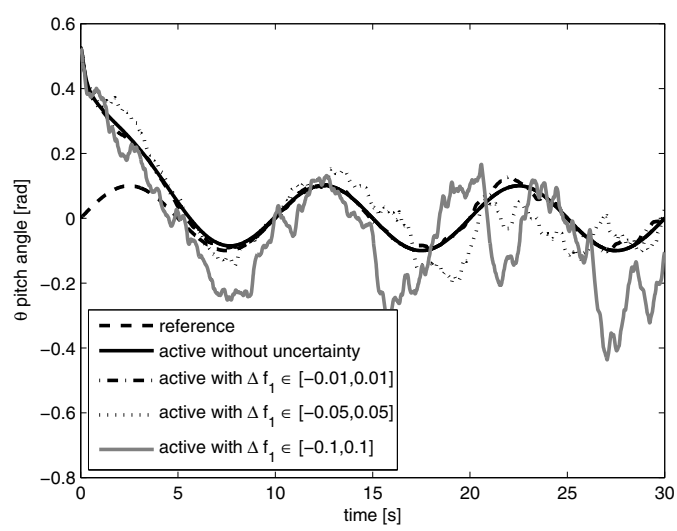

Fig. 8. Pitch angle response (active FTC without and with uncertainty, $f_{1}=0.7$ ).

\section{Acknowledgment}

This work has been funded by the Spanish MINECO through the project CICYT SHERECS (ref. DPI2011-26243), by the European Commission through the contract $i$-Sense (ref. FP7-ICT-2009-6-270428), by AGAUR through the contracts FI-DGR 2013 (ref. 2013FIB00218) and FI-DGR 2014 (ref. 2014FI_ B1 00172), and by the DGR of Generalitat de Catalunya (SAC group ref. 2014/SGR/374).

\section{References}

Abdullah, A. and Zribi, M. (2009). Model reference control of LPV systems, Journal of the Franklin Institute 346(9): 854-871.

Aguilar-Sierra, H., Flores, G., Salazar, S. and Lozano, R. (2014). Fault estimation for a quad-rotor MAV using a polynomial observer, Journal of Intelligent and Robotic Systems 73(1-4): 455-468.

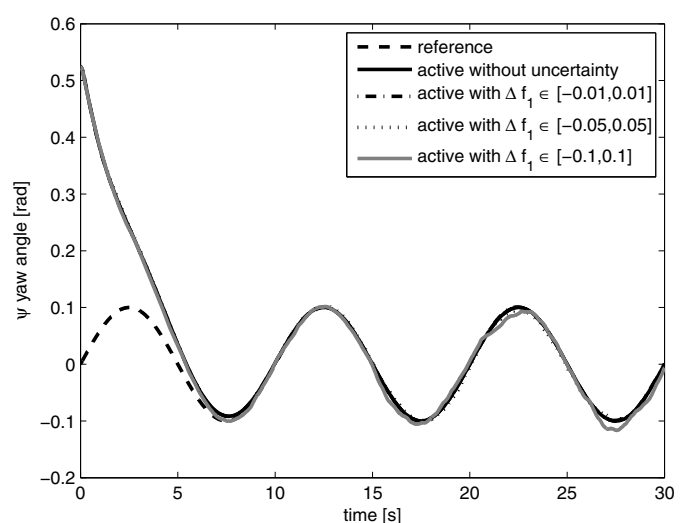

Fig. 9. Yaw angle response (active FTC without and with uncertainty, $f_{1}=0.7$ ).

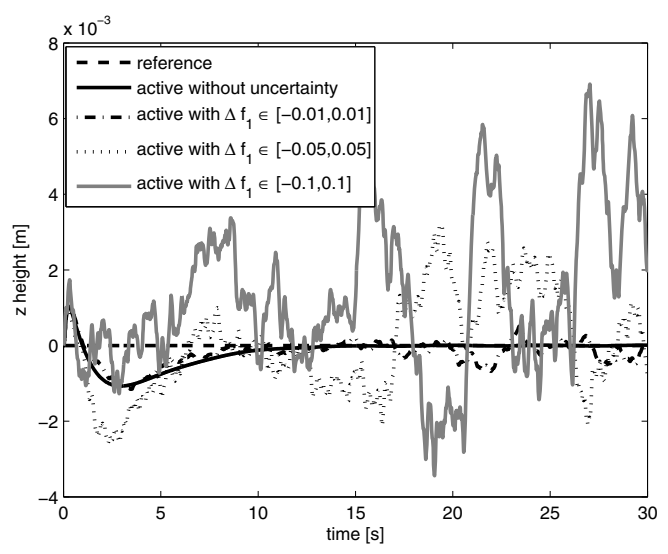

Fig. 10. Height response (active FTC without and with uncertainty, $\left.f_{1}=0.7\right)$.

Amoozgar, M., Chamseddine, A. and Zhang, Y. (2012). Fault-tolerant fuzzy gain-scheduled PID for a quadrotor helicopter testbed in the presence of actuator faults, Proceedings of the IFAC Conference on Advances in PID Control, Brescia, Italy, pp. 1-6.

Apkarian, P., Gahinet, P. and Becker, G. (1995). Self-scheduled $H_{\infty}$ control of linear parameter-varying systems: A design example, Automatica 31(9): 1251 - 1261.

Aranjo-Estrada, S., Liceaga-Castro, E. and Rodríguez-Cortés, H. (2009). Nonlinear motion control of a rotary wing vehicle powered by four rotors, Revista Ingeniería, Investigación y Tecnología 10(4): 373-383.

Benosman, M. (2010). A survey of some recent results on nonlinear fault tolerant control, Mathematical Problems in Engineering: 1-25, Article ID 586169, DOI: $10.1155 / 2010 / 586169$.

Bouabdallah, S., Murrieri, P. and Siegwart, R. (2004). Design and control of an indoor micro quadrotor, Proceedings of the IEEE International Conference on Robotics and Automation (ICRA), New Orleans, LA, USA, pp. 4393-4398. 


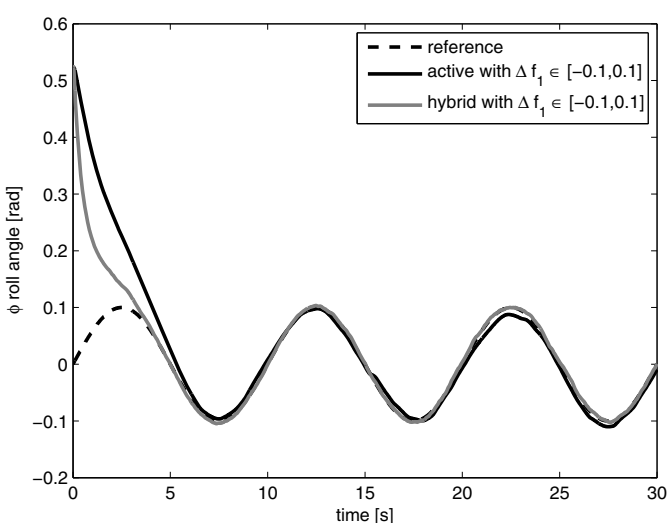

Fig. 11. Roll angle response (comparison between active FTC and hybrid FTC, $f_{1}=0.7$ ).

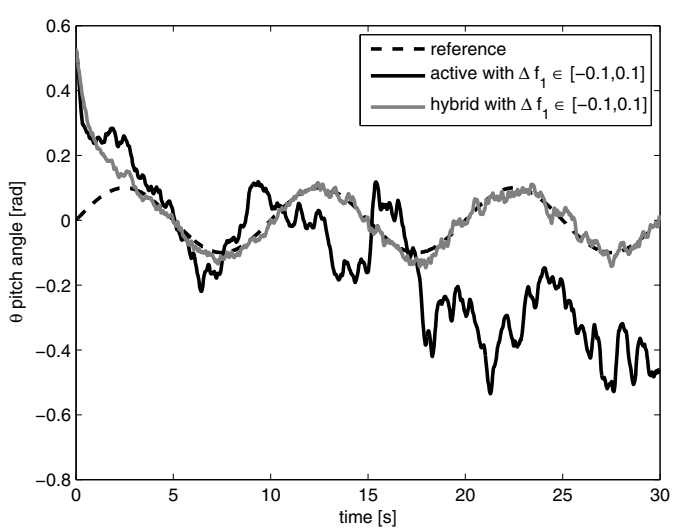

Fig. 12. Pitch angle response (comparison between active FTC and hybrid FTC, $f_{1}=0.7$ ).

Bresciani, T. (2008). Modelling, Identification and Control of a Quadrotor Helicopter, Master's thesis, Lund University, Lund.

Budiyono, A. and Sutarto, H. (2006). Linear parameter varying model identification for control of rotorcraft-based UAV, Proceedings of the 5th Taiwan-Indonesia Workshop on Aeronautical Science, Technology and Industry, Tainan, Taiwan, pp. 1-6.

Castillo, P., Lozano, R. and Dzul, A. (2005). Stabilization of a mini rotorcraft with four rotors, IEEE Control Systems Magazine 25: 45-55.

Cen, Z., Noura, H., Susilo, T.B. and Al Younes, Y. (2014). Robust fault diagnosis for quadrotor UAVs using adaptive Thau observer, Journal of Intelligent and Robotic Systems 73(1-4): 573-588.

Chamseddine, A., Sadeghzadeh, I., Zhang, Y. and Theilliol, D. (2012). Control allocation and re-allocation for a modified quadrotor helicopter against actuator faults, Proceedings of the 8th IFAC Symposium on Fault Detection, Supervision and Safety for Technical Processes (SAFEPROCESS), Mexico City, Mexico, pp. 247-252.

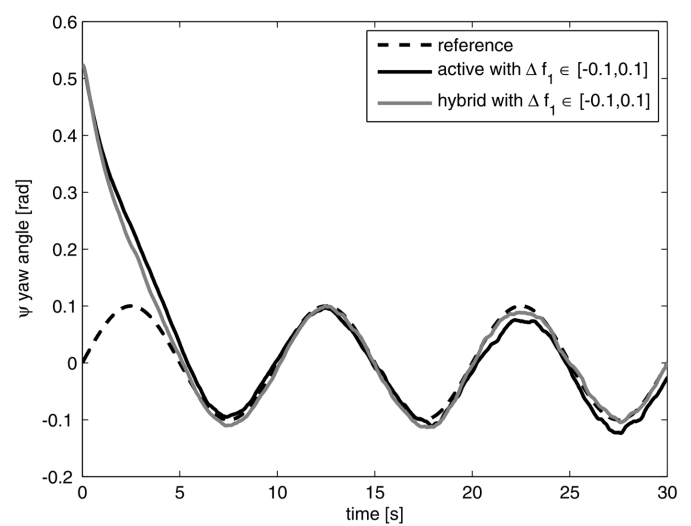

Fig. 13. Yaw angle response (comparison between active FTC and hybrid FTC, $f_{1}=0.7$ ).

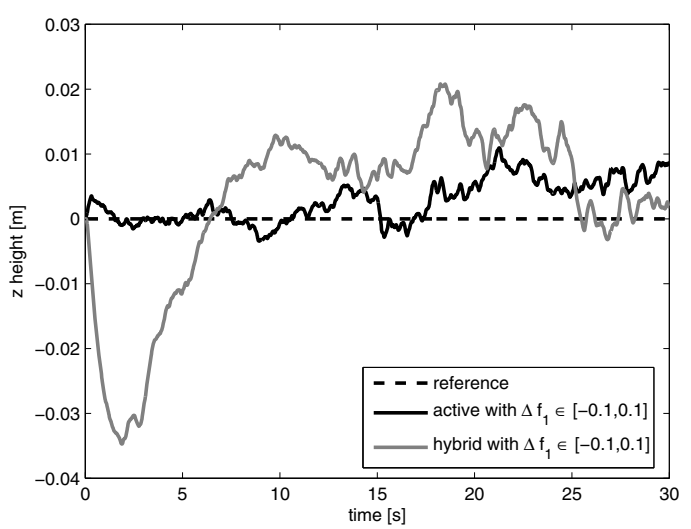

Fig. 14. Height response (comparison between active FTC and hybrid FTC, $\left.f_{1}=0.7\right)$.

Chamseddine, A., Zhang, Y., Rabbath, C., Fulford, C. and Apkarian, J. (2011). Model reference adaptive fault tolerant control of a quadrotor UAV, Proceedings of the AIAA Infotech Aerospace, St. Louis, MO, USA.

Chilali, M. and Gahinet, P. (1996). $H_{\infty}$ design with pole placement constraints: An LMI approach, IEEE Transactions on Automatic Control 41(3): 358-367.

Chowdhary, G., Frazzoli, E., How, J. and Liu, H. (2014). Nonlinear flight control techniques for unmanned aerial vehicles, in K.P. Valavanis and G.J. Vachtsevanos (Eds.), Handbook of Unmanned Aerial Vehicles, Springer, Houten.

Coleman, T., Branch, M.A. and Grace, A. (1999). Optimization Toolbox User's Guide, The Mathworks, Inc., Natick, MA.

Dydek, Z., Annaswamy, A. and Lavretsky, E. (2010a). Adaptive control of quadrotor UAVs in the presence of actuator uncertainties, Proceedings of the AIAA Infotech Aerospace, Atlanta, GA, USA, pp. 1-9.

Dydek, Z., Annaswamy, A. and Lavretsky, E. (2010b) Combined/composite adaptive control of a quadrotor UAV in the presence of actuator uncertainty, Proceedings of the AIAA Guidance, Navigation and Control Conference, Toronto, ON, Canada, pp. 1-10. 
Freddi, A., Lanzon, A. and Longhi, S. (2011). A feedback linearization approach to fault tolerance in quadrotor vehicles, Proceedings of the 18th IFAC World Congress, Milan, Italy, pp. 5413-5418.

Ghersin, A.S. and Sanchez-Peña, R.S. (2002). LPV control of a 6 DOF vehicle, IEEE Transactions on Control Systems Technology 10(6): 883-887.

Guenard, N., Hamel, T. and Mahony, R. (2008). A practical visual servo control for an unmanned aerial vehicle, IEEE Transactions on Robotics 24(2): 331-340.

Guerrero-Castellano, J.F., Téllez-Guzmán, J.J., Durand, S., Marchand, N., Alvarez-Muñoz, J.U. and González-Díaz, V.R. (2013). Attitude stabilization of a quadrotor by means of event-triggered nonlinear control, Journal of Intelligent and Robotic Systems 73(1-4): 123-135.

Hoffmann, G. and Waslander, S. (2008). Quadrotor helicopter trajectory tracking control, Proceedings of the AIAA Guidance, Navigation and Control Conference and Exhibit, Honolulu, HI, USA, pp. 1-14.

Horn, R.A. and Johnson, C.R. (1990). Matrix Analysis, Cambridge University Press, Cambridge.

Izadi, H., Gordon, B. and Zhang, Y. (2010). A data-driven fault tolerant model predictive control with fault identification, Proceedings of the 1st Conference on Control and FaultTolerant Systems (SYSTOL), Nice, France, pp. 732-737.

Izadi, H., Zhang, Y. and Gordon, B. (2011). Fault tolerant model predictive control of quad-rotor helicopters with actuator fault estimation, Proceedings of the 18th IFAC World Congress, Milan, Italy, pp. 6343-6348.

Jiang, J. and Yu, X. (2012). Fault-tolerant control systems: A comparative study between active and passive approaches, Annual Reviews in Control 36(1): 60-72.

Khebbache, H., Sait, B., Bounar, N. and Yacef, F. (2012). Robust stabilization of a quadrotor UAV in presence of actuator and sensor faults, International Journal of Instrumentation and Control Systems 2(2): 53-67.

Kwiatkowski, A., Boll, M.-T. and Werner, H. (2006). Automated generation and assessment of affine LPV models, Proceedings of the 45th IEEE Conference on Decision and Control, San Diego, CA, USA, pp. 6690-6695.

Landau, Y.D. (1979). Adaptive Control-The Model Reference Approach, Marcel Dekker, New York, NY.

Li, T., Zhang, Y. and Gordon, B. (2013). Passive and active nonlinear fault-tolerant control of a quadrotor UAV based on sliding mode control technique, Proceedings of the Institution of Mechanical Engineers, Part I: Journal of Systems and Control Engineering 227(1): 12-23.

Maki, M., Jiang, J. and Hagino, K. (2004). A stability guaranteed active fault-tolerant control system against actuator failures, International Journal of Robust and Nonlinear Control 14(12): 1061-1077.

Merheb, A.-R., Noura, H. and Bateman, F. (2013). Passive fault tolerant control of quadrotor UAV using regular and cascaded sliding mode control, Proceedings of the 2nd Conference on Control and Fault-Tolerant Systems (SYSTOL), Nice, France, pp. 330-335.
Milhim, A., Zhang, Y. and Rabbath, C. (2010). Gain scheduling based PID controller for fault tolerant control of a quadrotor UAV, Proceedings of AIAA Infotech Aerospace, Atlanta, GA, USA, pp. 1-13.

Mokhtari, A. and Benallegue, A. (2004). Dynamic feedback controller of Euler angles and wind parameter estimation for a quadrotor unmanned aerial vehicle, Proceedings of the IEEE International Conference on Robotics and Automation (ICRA), New Orleans, LA, USA, pp. 2359-2366.

Raffo, G.V., Ortega, M.G. and Rubio, F.R. (2010). An integral predictive/nonlinear $\mathcal{H}_{\infty}$ control structure for a quadrotor helicopter, Automatica 46(1): 29-39.

Rangajeeva, S. and Whidborne, J. (2011). Linear parameter varying control of a quadrotor, Proceedings of the 6th International Conference on Industrial and Information Systems (ICIIS), Kandy, Sri Lanka, pp. 483-488.

Rotondo, D., Nejjari, F. and Puig, V. (2012). Fault estimation and virtual actuator FTC approach for LPV systems, Proceedings of the 8th IFAC Symposium on Fault Detection, Supervision and Safety for Technical Processes (SAFEPROCESS), Mexico City, Mexico, pp. 824-829.

Rotondo, D., Nejjari, F. and Puig, V. (2013a). Fault tolerant control design for polytopic uncertain LPV systems, Proceedings of the 21st Mediterranean Conference on Control and Automation, Platanias-Chania, Crete, Greece, pp. 66-72.

Rotondo, D., Nejjari, F. and Puig, V. (2013b). Passive and active FTC comparison for polytopic LPV systems, Proceedings of the 12th European Control Conference, Zurich, Switzerland, pp. 2951-2956.

Rotondo, D., Nejjari, F., Torren, A. and Puig, V. (2013c). Fault tolerant control design for polytopic uncertain LPV systems: Application to a quadrotor, Proceedings of the 2nd Conference on Control and Fault-Tolerant Systems (SYSTOL), Nice, France, pp. 643-648.

Rotondo, D., Nejjari, F. and Puig, V. (2014). Model reference quasi-LPV control of a quadrotor UAV, IEEE Multiconference on Systems and Control, Antibes, France, pp. 736-741.

Sadeghzadeh, I., Mehta, A. and Zhang, Y. (2011a). Fault/damage tolerant control of a quadrotor helicopter UAV using model reference adaptive control and gain-scheduled PID, Proceedings of the AIAA Guidance, Navigation and Control Conference, Portland, OR, USA.

Sadeghzadeh, I., Mehta, A., Zhang, Y. and Rabbath, C. (2011b). Fault-tolerant trajectory tracking control of a quadrotor helicopter using gain-scheduled PID and model reference adaptive control, Proceedings of the Annual Conference of the Prognostics and Health Management Society, Montreal, QC, Canada, pp. 1-10.

Serirojanakul, A. and Wongaisuwan, M. (2012). Optimal control of quad-rotor helicopter using state feedback LPV method, Proceedings of the 9th International Conference on Electrical Engineering/Electronics, Computer Telecommunications and Information Technology (ECTI-CON), Hua Hin, Thailand, pp. 1-4. 
Shamma, J.S. (2012). An overview of LPV systems, in J. Mohammadpour and C. Scherer (Eds.), Control of Linear Parameter Varying Systems with Applications, Springer, New York, NY, pp. 3-26.

Sharifi, F., Mirzaei, M., Gordon, B. and Zhang, Y. (2010). Fault tolerant control of a quadrotor UAV using sliding mode control, Proceedings of the 1st Conference on Control and Fault-Tolerant Systems (SYSTOL), Nice, France, pp. 239-244.

Staroswiecki, M., Berdjag, D., Jiang, B. and Zhang, K. (2009). PACT: A passive/active approach to fault tolerant stability under actuator outages, Proceedings of the Joint 48th IEEE Conference on Decision and Control and the 28th Chinese Control Conference, Shanghai, China, pp. 7819-7824.

Staroswiecki, M., Zhang, K., Berdjag, D. and Abbas-Turki, M. (2012). Reducing the reliability over-cost in reconfiguration-based fault tolerant control under actuator faults, IEEE Transactions on Automatic Control 57(12): 3181-3186.

Yu, B., Zhang, Y., Minchala, I. and Qu, Y. (2013). Fault-tolerant control with linear quadratic and model predictive control techniques against actuator faults in a quadrotor UAV, Proceedings of the 2nd Conference on Control and FaultTolerant Systems (SYSTOL), Nice, France, pp. 661-666.

Yu, X. and Jiang, J. (2012). Hybrid fault-tolerant flight control system design against partial actuator failures, IEEE Transactions on Control Systems Technology 20(4): 871-886.

Zhang, X. and Zhang, Y. (2010). Fault tolerant control for quadrotor UAV by employing Lyapunov-based adaptive control approach, Proceedings of the AIAA Guidance, Navigation and Control Conference, Toronto, ON, Canada.

Zhang, X., Zhang, Y., Su, C.-Y. and Feng, Y. (2010). Fault-tolerant control for quad-rotor UAV via backstepping approach, Proceedings of the 48th AIAA Aerospace Sciences Meeting, Orlando, FL, USA.

Zhang, Y. and Jiang, J. (2008). Bibliographical review on reconfigurable fault-tolerant control systems, Annual Reviews in Control 32(2): 229-252.

Zhang, Y. M., Chamseddine, A., Rabbath, C. A., Gordon, B. W., Su, C.-Y., Rakheja, S., Fulford, C., Apkarian, J. and Gosselin, P. (2013). Development of advanced FDD and FTC techniques with application to an unmanned quadrotor helicopter testbed, Journal of the Franklin Institute 350(9): 2396-2422.

Zhaohui, C. and Noura, H. (2013). A composite fault tolerant control based on fault estimation for quadrotor UAVs, Proceedings of the 8th IEEE Conference on Industrial Electronics and Applications (ICIEA), Melbourne, Australia, pp. 236-241.

Zhou, Q.-L., Zhang, Y., Rabbath, C. and Theilliol, D. (2010). Design of feedback linearization control and reconfigurable control allocation with application to quadrotor UAV, Proceedings of the 1st Conference on Control and Fault-Tolerant Systems (SYSTOL), Nice, France, pp. 371-376.

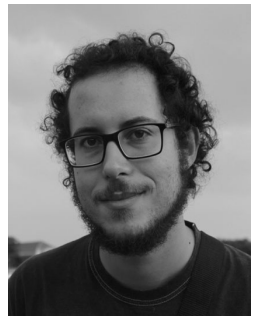

Damiano Rotondo was born in Francavilla Fontana, Italy, in 1987. He received the B.Sc. degree (with honors) from the Second University of Naples, Italy, and the M.Sc. degree (with honors) from the University of Pisa, Italy, in 2008 and 2011, respectively. Since 2011, he has been a Ph.D. student at the Research Center for Supervision, Safety and Automatic Control (CS2AC), Technical University of Catalonia (UPC), Barcelona, Spain. His main research interests include gain-scheduled control systems, fault detection and isolation, and fault tolerant control of dynamic systems. He has publishec several papers in international conference proceedings and scientific journals.

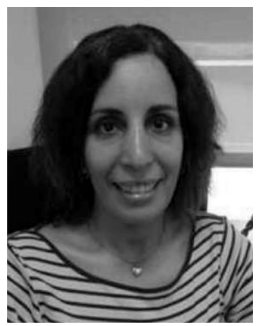

Fatiha Nejjari received the M.Sc. in physics from Hassan II University, Casablanca, Morocco, in 1993 and the Ph.D. in automatic control from Cadi Ayyad University, Marrakech, Morocco, in 1997. She is currently an associate professor with the Department of Automatic Control, Universitat Politécnica de Catalunya (UPC). She is also a member of the Advanced Control Systems (SAC) research group of the Research Center for Supervision, Safety and Automatic Control (CS2AC) at UPC. Her main research areas include LPV control, fault detection and isolation, and fault tolerant control of dynamic systems. She has published several papers in journals and international conferences and participated in several European projects and networks related to these topics.

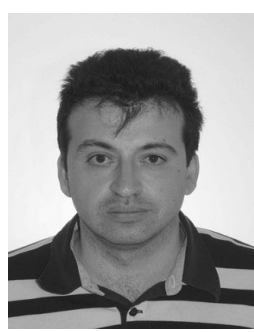

Vicenç Puig was born in Girona, Spain, in 1969, received the Ph.D. degree in control engineering in 1999 and the telecommunications engineering degree in 1993, both from Universitat Politécnica de Catalunya (UPC), Barcelona, Spain. He is currently an associate professor of automatic control and the leader of the Advanced Control Systems (SAC) research group of the Research Center for Supervision, Safety and Automatic Control (CS2AC) at UPC. His main research interests are fault detection, isolation of fault-tolerant control of dynamic systems. He has been involved in several European projects and networks, and has published many papers in international conference proceedings and scientific journals.
Received: 18 January 2014

Revised: 22 April 2014 